\title{
Detection of a Markovian Target with Optimization of the Search Efforts under Generalized Linear Constraints
}

Frédéric Dambreville, Jean-Pierre Le Cadre

\section{$\mathbf{N}^{\circ} 3815$}

Novembre 1999

THÈME 3 



\title{
RINRIA
}

\section{Detection of a Markovian Target with Optimization of the Search Efforts under Generalized Linear Constraints}

\author{
Frédéric Dambreville, Jean-Pierre Le Cadre \\ Thème 3 - Interaction homme-machine, \\ images, données, connaissances \\ Projet Vista
}

Rapport de recherche $\mathrm{n}^{\circ} 3815$ - Novembre 1999 - 38 pages

\begin{abstract}
This report deals with search for a target following a Markovian movement or a conditionally deterministic motion. The problem is to allocate the search efforts, when search resources renew with generalized linear constraints. The model obtained is extended to resource mixing management. New optimality equations of de Guenin's style are obtained. Practically, the problem is solved by using an algorithm derived from the F.A.B. method.
\end{abstract}

Key-words: Search theory, Detection, Optimization, Markovian movement, Sensor management

(Résumé : tsvp) 


\section{Détection d'une cible markovienne avec optimisation des efforts de recherche sous contraintes linéaires généralisées}

Résumé : Ce rapport concerne la recherche d'une cible suivant un mouvement markovien ou conditionellement déterministe. Le problème est d'allouer un effort de recherche, lorsque les ressources de détection disponibles se renouvellent suivant des contraintes linéaires généralisées. Le modèle obtenu est étendu à la gestion de mélanges de ressources de détection. De nouvelles équations de de Guenin sont obtenues. Pratiquement, le problème est résolu en utilisant un algorithme dérivé de la méthode F.A.B.

Mots-clé : Théorie de la recherche, Détection, Optimisation, Mouvement markovien, Gestion de capteurs 


\section{Introduction}

Search theory is the discipline which treats the problem of how best to search for an object when the amount of searching efforts is limited and only probabilities of the object's possible position are given. Search theory came into being during World War II with the work of B.O. Koopman and his colleagues [1] in the Antisubmarine Warfare Operations Research Group (ASWORG). Since that time, search theory has grown to be a major discipline within the field of operations research. An important literature has been devoted to this subject, interested reader may consult various extensive surveys [4], introductory texts [3], and books [5], [6], [7], [8].

The situation is characterized by three data:

(i) the probabilities of the searched object (the "target") being in various possible locations;

(ii) the local detection probability that a particular amount of local search effort should detect the target;

(iii) the total amount of searching effort available. The problem is to find the optimal distribution of this total effort, i.e. which maximizes the probability of detection.

Solving such problem requires to optimize a huge amount of variable (3600 to 12600 for our examples). Decisive improvements have been made for finding search strategies that maximize the probability of detecting a moving target within a fixed amount of time periods. In particular, Brown has proposed an iterative algorithm in which the motion space and the time frame have been discretized, and the search effort available for each period is infinitely divisible between the grid cells of the targegt motion space. In this approach, the search effort available for each period is bounded above by a constant that does not depend on the allocations made during any other periods.

However, even if the general formalism of search theory will be of constant use subsequently, we shall consider now a specific problem. In the framework of "classical" search problems, the amount of search effort available at each period is bounded above by a fixed and known value. However, for a multi-period search, the final result (the global probability of detection) is tightly related to the sequence

of successive search amounts. Thus, optimizing the sequence of search amounts is

$\mathrm{RR} \mathrm{n}^{\circ} 3815$ 
quite challenging. However, it is not possible to optimize separately the sequence of search amounts and the search plans (i.e. the distribution of elementary search efforts). Here, we shall consider general constraints relative to this sequence of search amounts. These constraints may take into account specifications relative to the renewal of search resources (see section 3) as well as general operational requirements. It is worth stressing that associated optimization problem has not be fully addressed in the literature.

The general optimality equations are derived by means of a method largely inspired from classical search theory (namely de Guenin's equations), though they are considerably more complicated (see section 2.2). An original algorithmic approach has been used for solving the optimization problem. It combines theoretical results of section 2.2 with a study of the differential changes of the non-detection probability (see section 4). In order to render the problem feasible, the Markovian hypothesis (relative to the target motion) is instrumental, allowing us to use the Brown's implementation (see section 4 and 5). Various extensions will then be considered, namely extension to mixed resources (section 5) and inequality constraints (section 6). Finally, our methods are illustrated by simulation results (see section 7).

\section{The search problem}

\subsection{One period search}

The problem is to detect a target $x$, lying in a space $E$, and whose location is characterized by a (known) density $\alpha(x)$. To make this detection, a limited amount of search resource $\phi$ is available. This (total) search effort may be distributed along the whole space $E$. To describe the distribution of the search effort, we denote $\varphi(x)$ the search density allocated to $x \in E$. The limitations on search resource inferred by $\phi$ yields the following condition on the search effort distribution $\varphi(x)$ :

$$
\int_{E} \varphi(x) d x \leq \phi
$$

Under the assumption that the target's location is $x$ and when the local search effort is $\varphi(x)$, we call $p_{x}(\varphi(x))$ the probability not to detect the target (this probability may depend upon $x$ ). For $x$ fixed, $p_{x}$ decreases with the effort used and then $p_{x}^{\prime}<0$. We suppose the detection follows the rule of decreasing return, so that $p_{x}^{\prime}$ increases

strictly with $\phi$. According to these notations, our problem is to find the search effort 
$\varphi$ under the condition (1) in order to minimize $P_{n d}(\varphi)$, the global probability of non detection:

$$
P_{n d}(\varphi)=\int_{E} \alpha(x) p_{x}(\varphi(x)) d x .
$$

As the probability of non detection decreases with the increase of search effort, the condition (1) becomes (3) :

$$
\int_{E} \varphi(x) d x=\phi
$$

From (2), (3) and from the positivity of density $\varphi$, the de Guenin's equations [9] are obtained (see equation (4)). They give optimality conditions on $\varphi$, scaled by a scalar term $\eta \leq 0$, i.e. :

$$
\left\{\begin{aligned}
\alpha(x) p_{x}^{\prime}(\varphi(x)) & =\eta & & \text { if } \alpha(x)>\eta / p_{x}^{\prime}(0) \\
\varphi(x) & =0 & & \text { else }
\end{aligned}\right.
$$

Using inversion of $p_{x}^{\prime}$ in (4), function $\varphi_{\eta}$ is obtained. Since $p_{x}^{\prime}$ is strictly monotonic increasing, $\varphi_{\eta}$ increases uniformly (i.e. for each $x \in E$ ) with $\eta$ and $\int_{E} \varphi_{\eta}(x) d x$ increases. Then $\varphi_{\eta}$ will satisfy (3) for only one value of $\eta$. Once the good value of $\eta$ is obtained (e.g. by means of an elementary dichotomy), the optimal function $\varphi$ is deduced ( $p_{x}^{\prime}$ is invertible) from equation (4).

This very fast method, tracing back to the seminal work of B.O. Koopman and J. de Guenin, has been extended by S.S. Brown to deal with multi-period search for a Markovian moving target. It will be of constant use subsequently.

\subsection{Multi-period search}

Our objective is to detect at one or more time-period a target moving in a given space $E$ (assuming stationarity for each period). The detection is done within $T$ time-periods and the search ends after the first detection. We define $\vec{x}=\left(x_{1}, \ldots, x_{T}\right)$ the position of the target during the time-periods $1,2, \ldots, T$. We assume that the target motion is probabilistic and Markovian. Because of the Markovian property, the probabilistic density $\alpha(\vec{x})=\alpha\left(x_{1}, \ldots, x_{T}\right)$ of the target trajectory may be written as a product of elementary densities, i.e. :

$$
\alpha(\vec{x})=\prod_{k=1}^{T-1} \alpha_{k, k+1}\left(x_{k}, x_{k+1}\right) .
$$

$\mathrm{RR} \mathrm{n}^{\circ} 3815$ 
For each time-period $k$ a given amount of search effort $\phi_{k}$ is available. It may be distributed along the search space $E$. The (local) search effort, applied to the point $x_{k} \in E$ at time $k$, is denoted $\varphi_{k}\left(x_{k}\right)$. So, at each time-period, the following (equality) constraint (6) is commonly considered in the search theory literature:

$$
\forall k \in\{1, \ldots, T\}, \int_{E} \varphi_{k}(x) d x=\phi_{k} .
$$

We call $p_{k, x_{k}}\left(\varphi_{k}\left(x_{k}\right)\right)$ the probability not to detect the target within the time-period $k$, when its location is indeed $x_{k}$. We still assume that the detection follows the law of diminishing return. Thus for $x_{k}$ fixed, $p_{k, x_{k}}^{\prime}<0$ and $p_{k, x_{k}}^{\prime}$ is strictly increasing.

The problem is then to find the functions $\varphi_{k}$ in order to minimize $\mathbf{P}_{n d}(\varphi)$ the global probability of non detection, under the constraint (6). Since the elementary detections are independent, $\mathbf{P}_{n d}(\varphi)$ stands as follows:

$$
\mathbf{P}_{n d}(\varphi)=\int_{E^{T}} \alpha(\vec{x}) \prod_{k=1}^{k=T} p_{k, x_{k}}\left(\varphi_{k}\left(x_{k}\right)\right) \prod_{k=1}^{k=T} d x_{k} .
$$

For a particular time-period $\kappa, \mathbf{P}_{n d}(\varphi)$ can also be written:

$$
\mathbf{P}_{n d}(\varphi)=\int_{E} \beta_{\kappa}^{\varphi}\left(x_{\kappa}\right) p_{\kappa, x_{\kappa}}\left(\varphi_{\kappa}\left(x_{\kappa}\right)\right) d x_{\kappa}
$$

where:

$$
\beta_{\kappa}^{\varphi}\left(x_{\kappa}\right)=\int_{E^{T-1}} \alpha(\vec{x}) \prod_{1 \leq k \leq T}^{k \neq \kappa}\left(p_{k, x_{k}}\left(\varphi_{k}\left(x_{k}\right)\right) d x_{k}\right) .
$$

This shows that, when the search efforts are fixed for all the time-periods, except for a given one denoted $\kappa$, the optimization problem may be solved as the following 1-period de Guenin's problem:

$$
\begin{aligned}
& \text { Minimize: } P_{n d}\left(\varphi_{\kappa}\right)=\int_{E} \beta_{\kappa}^{\varphi}(x) p_{\kappa, x}\left(\varphi_{\kappa}(x)\right) d x, \\
& \text { subject to: } \int_{E} \varphi_{\kappa}(x) d x=\phi_{\kappa} \text { and } \varphi_{\kappa} \geq 0 .
\end{aligned}
$$

Then, the following de Guenin's conditions are obtained and inverted by the algorithm described in section 2.1:

$$
\left\{\begin{array}{cl}
\beta_{\kappa}^{\varphi}(x) p_{\kappa, x}^{\prime}\left(\varphi_{\kappa}(x)\right)=\eta_{\kappa} & \text { if } \beta_{\kappa}^{\varphi}(x)>\eta_{\kappa} / p_{\kappa, x}^{\prime}(0) \\
\varphi_{\kappa}(x)=0 & \text { else }
\end{array}\right.
$$


Brown's algorithm follows these general guidelines. The distributions of the search efforts are successively optimized for each time-period (See Figure 1), the other ones being fixed. Convergence requires only a few iterations. A fundamental ingredient

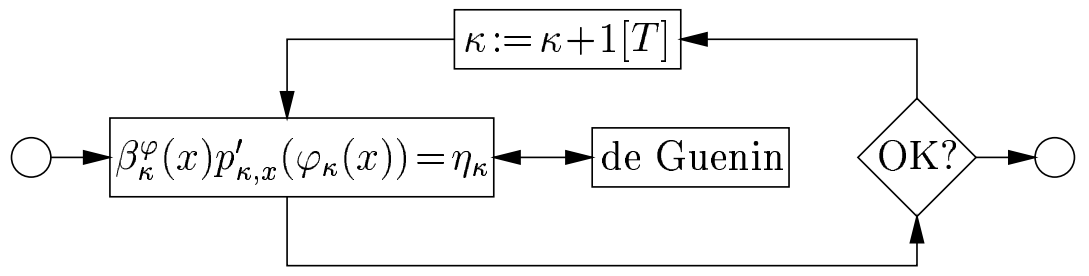

Figure 1: Algorithm for multi-period search

of this algorithm is to use basically the Markovian assumption relative to $\alpha$, so as to drastically reduce the computation requirements for the integral (8), (Forward And Backward algorithm [10], [11]). As we shall see later, this idea will be instrumental for the development of a feasible algorithm for solving the search problem with generalized constraints.

Until now, the constraints we considered were directly related to the values of $\phi_{k}$. The aim of this article is to generalize the multi-period search to more flexible constraints. This includes especially simple cases of resource renewal.

\section{Generalized constraints}

As seen previously, the algorithm of Brown supposes the time-splitting $\phi$ of the contraints to be known. For example, Brown's algorithm does not know how to share (optimally) the global amount of search resources between each period of search. First, we shall consider a simple linear framework allowing us to deal with a great variety of resource time-sharing and to solve efficiently the related optimization problems.

\subsection{Resource time-sharing specifications}

A general formulation of resource time-sharing will be built below and illustrated by two examples. It will be of constant use subsequently.

$\mathrm{RR} \mathrm{n}^{\circ} 3815$ 


\subsubsection{Splitting non renewable resources}

We dispose of a total amount of search resources equal to $N$, one time-period only usable. As we want to optimize the probability of detection within the $T$ periods, we have to split $N$ into $T$ period resources $\phi_{k}$ so that $\sum_{k=1}^{T} \phi_{k}=N$, or equivalently:

$$
A \phi=\psi,
$$

where $A$ is the matrix $A_{N R}=(1 \ldots 1)$ with $T$ columns, $\phi$ is the $T$-dimensional vector of search efforts and $\psi$ is the 1 -dimensional vector $\psi_{N R}=(N)$. In section 7 , we will refer to this matrix as $A_{N R}$ and to this vector as $\psi_{N R}$.

\subsubsection{Splitting renewable resources}

Assume now that we have a search amount $N$ renewable after some time-periods (time for replenishment, for moving, etc.). For example we can think that resources renew after two periods so that the same resource cannot be used simultaneously in two consecutive periods. That means for two following periods $k$ and $k+1$ the relation $\phi_{k}+\phi_{k+1}=N$. These relations are equivalent to the following linear constraints:

$$
A \phi=\psi,
$$

where $A$ is the pseudo-diagonal matrix $A_{R}=\left(a_{R}(i, j)\right)_{i, j}$ with $T-1$ rows and $T$ columns defined by:

$$
\left\{\begin{array}{l}
\forall i \in\{1, \ldots, T-1\}, a_{R}(i, i)=a_{R}(i, i+1)=1 \\
a_{R}(i, j)=0 \text { else. }
\end{array}\right.
$$

and $\psi$ is the vector $\psi_{R}=(N \ldots N)^{t}$ with $T-1$ components. In section 7 , we will refer to this matrix and its corresponding constraint vector as $A_{R}$ and $\psi_{R}$.

\subsubsection{The general optimization problem}

More generally, the linear formulation:

$$
A \phi=\psi
$$

of period-sharing constraints seems sufficiently versatile to handle a great variety of resource allocation problems. 
Using the above notations, our objective is still to minimize the non detection probability $\mathbf{P}_{n d}(\varphi)$ defined in (7), but now under the following generalized constraints:

$$
A\left(\int_{E} \varphi_{k}(x) d x\right)_{1 \leq k \leq T}=\left(\psi_{j}\right)_{1 \leq j \leq \Theta},
$$

where $A$ represents the matrix of resource renewal. In the general case, the $A$ matrix is rectangular, the column number being greater than the row one. In equation (15), $\psi$ is the vector of resource constraints. Its dimension is $\Theta$. Furthermore, a reasonable hypothesis is that $A X=\psi$ admits at least one solution.

\subsection{Generalized de Guenin's equations}

We shall now prove that any search effort $\varphi$ minimizing $\mathbf{P}_{n d}(\varphi)$ under constraints (15) will satisfy optimality equations rather analogous to the de Guenins's equations. The following optimality conditions extend the classical ones (see equation (4)) to the generalized constraints.

Property 1 Let $\varphi=\left(\varphi_{k}\right)_{1 \leq k \leq T}$ be an optimal solution. Suppose $\forall k, \varphi_{k} \neq 0$, then ${ }^{1}$ :

1. There is a vector $\mu=\left(\mu_{k}\right)_{1 \leq k \leq \Theta}$ such that, for each element $\vec{a}$ of $E^{T}$ verifying $\forall k, \varphi_{k}\left(a_{k}\right)>0$, we have:

$$
\left(p_{k, a_{k}}^{\prime}\left(\varphi_{k}\left(a_{k}\right)\right) \beta_{k}^{\varphi}\left(a_{k}\right)\right)_{1 \leq k \leq T}=A^{t} \mu
$$

2. Let $\eta=A^{t} \mu$. Let $\kappa \in\{1, \ldots, T\}$ and $a_{\kappa} \in E$, then:

$$
\beta_{\kappa}^{\varphi}\left(a_{\kappa}\right) \leq \frac{\eta_{\kappa}}{p_{\kappa, a_{\kappa}}^{\prime}(0)} \Longleftrightarrow \varphi_{\kappa}\left(a_{\kappa}\right)=0
$$

Compared with (10), (16) shows interdependence between the optimality equations. Consequently, they cannot be solved by a direct application of the Brown's algorithm.

\footnotetext{
${ }^{1} A^{t}$ denotes the transpose of the $A$ matrix.
} 
Proof of 1. Let $\varphi$ be an optimal solution. We shall consider (small) variations of $\varphi$, both horizontally and vertically, satisfying the constraints. Each of these type of variations will give a differential property, which the optimal solution $\varphi$ must satisfy. The property (16) will be proved by gathering these sub-properties.

Let $\kappa \in\{1, \ldots, T\}$ and let $a_{\kappa}$ and $b_{\kappa}$ be two elements of $E$ satisfying $\varphi_{\kappa}\left(a_{\kappa}\right)>0$ and $\varphi_{\kappa}\left(b_{\kappa}\right)>0$. We consider $d t$ an infinitesimal scalar variation. The search distribution $\varphi$ is changed to yield $\tilde{\varphi}$ by the following procedure:

$$
\left\{\begin{array}{l}
\tilde{\varphi}_{\kappa}\left(a_{\kappa}\right)=\varphi_{\kappa}\left(a_{\kappa}\right)+d t, \tilde{\varphi}_{\kappa}\left(b_{\kappa}\right)=\varphi_{\kappa}\left(b_{\kappa}\right)-d t \\
\tilde{\varphi}_{k}\left(x_{k}\right)=\varphi_{k}\left(x_{k}\right) \text { else }
\end{array}\right.
$$

As $\varphi$ is such that constraint (15) is fulfilled, we easily prove that $\tilde{\varphi}$ satisfies it too. As $\varphi_{\kappa}\left(a_{\kappa}\right)>0$ and $\varphi_{\kappa}\left(b_{\kappa}\right)>0, \tilde{\varphi}$ still satisfies to the positivity density constraint $\tilde{\varphi} \geq 0$, as long as $d t$ is sufficiently small. With $\tilde{\varphi}$ satisfying the optimization constraints and $\varphi$ being optimal, we must have $\mathbf{P}_{n d}(\varphi) \leq \mathbf{P}_{n d}(\tilde{\varphi})$. Using the formulation (8), we make a first order expansion of $\mathbf{P}_{n d}(\tilde{\varphi})$ on $d t$ and simplify each member of the obtained inequality, thus yielding:

$$
0 \leq\left(p_{\kappa, a_{\kappa}}^{\prime}\left(\varphi_{\kappa}\left(a_{\kappa}\right)\right) \beta_{\kappa}^{\varphi}\left(a_{\kappa}\right)-p_{\kappa, b_{\kappa}}^{\prime}\left(\varphi_{\kappa}\left(b_{\kappa}\right)\right) \beta_{\kappa}^{\varphi}\left(b_{\kappa}\right)\right) d t .
$$

As the sign of $d t$ is arbitrary, we then deduce:

$$
0=\left(p_{\kappa, a_{\kappa}}^{\prime}\left(\varphi_{\kappa}\left(a_{\kappa}\right)\right) \beta_{\kappa}^{\varphi}\left(a_{\kappa}\right)-p_{\kappa, b_{\kappa}}^{\prime}\left(\varphi_{\kappa}\left(b_{\kappa}\right)\right) \beta_{\kappa}^{\varphi}\left(b_{\kappa}\right)\right) .
$$

The only constraint upon the choice of $a_{\kappa}$ and $b_{\kappa}$ is that $\varphi$ must be strictly positive on theses points. The following lemma has thus been obtained:

Lemma 1 Let $\varphi$ be an optimal solution. Then for all $\kappa \in\{1, \ldots, T\}$, there is a constant $c_{\kappa}$ so that:

$$
\forall x \in E, \varphi_{\kappa}(x)>0 \Rightarrow p_{\kappa, x}^{\prime}\left(\varphi_{\kappa}(x)\right) \beta_{\kappa}^{\varphi}(x)=c_{\kappa} .
$$

Now, let $\vec{a} \in E^{T}$ such that $\forall k, \varphi_{k}\left(a_{k}\right)>0$. Let $d t$ be an infinitesimal scalar and $\lambda$ any vector of $\operatorname{ker} A$. We define $d \phi=d t \lambda$ and denote $d \phi_{k}$ the $k$-th component of $d \phi$. We then build $\check{\varphi}$ a perturbation of $\varphi$ by the following procedure:

$$
\left\{\begin{array}{l}
\forall k \in\{1, \ldots, T\}, \check{\varphi}_{k}\left(a_{k}\right)=\varphi_{k}\left(a_{k}\right)+d \phi_{k} \\
\check{\varphi}_{k}(x)=\varphi_{k}(x) \text { else }
\end{array}\right.
$$


Since $\forall k, \varphi_{k}\left(a_{k}\right)>0$, the perturbed search distribution $\check{\varphi}$ remains positive as long as $d t$ is sufficiently small. Furthermore, we have:

$$
A\left(\int_{E} \check{\varphi}_{k}(x) d x\right)_{k}=A\left(\int_{E} \varphi_{k}(x) d x\right)_{k}+A d \phi .
$$

As $A d \phi=d t(A \lambda)=0$ and since $\varphi$ satisfies the constraint (15), this condition is fulfilled for $\check{\varphi}$. As $\varphi$ is optimal, we deduce that $\mathbf{P}_{n d}(\varphi) \leq \mathbf{P}_{n d}(\check{\varphi})$. Using the formulation (8), we make a first order expansion of $\mathbf{P}_{n d}(\check{\varphi})$ relatively to $d t$ and we simplify each member of the obtained inequality, thus yielding:

$$
0 \leq(d t \lambda)^{t}\left(p_{k, a_{k}}^{\prime}\left(\varphi_{k}\left(a_{k}\right)\right) \beta_{k}^{\varphi}\left(a_{k}\right)\right)_{1 \leq k \leq T} .
$$

The sign of $d t$ being arbitrary, the following equality holds:

$$
\lambda^{t}\left(p_{k, a_{k}}^{\prime}\left(\varphi_{k}\left(a_{k}\right)\right) \beta_{k}^{\varphi}\left(a_{k}\right)\right)_{1 \leq k \leq T}=0 .
$$

This equality holding true for any $\lambda \in \operatorname{ker} A$, we obtain:

$$
\left(p_{k, a_{k}}^{\prime}\left(\varphi_{k}\left(a_{k}\right)\right) \beta_{k}^{\varphi}\left(a_{k}\right)\right)_{1 \leq k \leq T} \in(\operatorname{ker} A)^{\perp} .
$$

Now, consider the constants $c_{\kappa}$ defined by the lemma 1 . From equation (25), we infer that $\left(c_{k}\right)_{1 \leq k \leq T} \in(\operatorname{ker} A)^{\perp}$. As $(\operatorname{ker} A)^{\perp}=\operatorname{Im}\left(A^{t}\right)$, there is a $\mu$ vector such that $\left(c_{k}\right)_{1 \leq k \leq T}=A^{\bar{t}} \mu$. From lemma 1 the property (16) then follows.

Proof of 2. Suppose that $\varphi_{\kappa}\left(a_{\kappa}\right)=0$. Let us consider the horizontal variation of $\varphi$ as it has been defined in the precedent proof. That is, we choose $d t \geq 0$ (since $\left.\varphi_{\kappa}\left(a_{\kappa}\right)=0\right), b_{\kappa}$ so that $\varphi_{\kappa}\left(b_{\kappa}\right)>0$ and build $\tilde{\varphi}$ :

$$
\left\{\begin{array}{l}
\tilde{\varphi}_{\kappa}\left(a_{\kappa}\right)=\varphi_{\kappa}\left(a_{\kappa}\right)+d t, \tilde{\varphi}_{\kappa}\left(b_{\kappa}\right)=\varphi_{\kappa}\left(b_{\kappa}\right)-d t \\
\tilde{\varphi}_{k}\left(x_{k}\right)=\varphi_{k}\left(x_{k}\right) \text { else }
\end{array}\right.
$$

Then, the optimality of $\varphi$ leads to the inequality $\mathbf{P}_{n d}(\varphi) \leq \mathbf{P}_{n d}(\tilde{\varphi})$, which can be rewritten:

$$
0 \leq\left(p_{\kappa, a_{\kappa}}^{\prime}\left(\varphi_{\kappa}\left(a_{\kappa}\right)\right) \beta_{\kappa}^{\varphi}\left(a_{\kappa}\right)-p_{\kappa, b_{\kappa}}^{\prime}\left(\varphi_{\kappa}\left(b_{\kappa}\right)\right) \beta_{\kappa}^{\varphi}\left(b_{\kappa}\right)\right) d t
$$

As the sign of $d t$ is positive, we only deduce the inequality:

$$
\left(p_{\kappa, a_{\kappa}}^{\prime}\left(\varphi_{\kappa}\left(a_{\kappa}\right)\right) \beta_{\kappa}^{\varphi}\left(a_{\kappa}\right)-p_{\kappa, b_{\kappa}}^{\prime}\left(\varphi_{\kappa}\left(b_{\kappa}\right)\right) \beta_{\kappa}^{\varphi}\left(b_{\kappa}\right)\right) \geq 0 .
$$

$\mathrm{RR} \mathrm{n}^{\circ} 3815$ 
Now, invoking the first part of Property 1 yields:

$$
p_{\kappa, b_{\kappa}}^{\prime}\left(\varphi_{\kappa}\left(b_{\kappa}\right)\right) \beta_{\kappa}^{\varphi}\left(b_{\kappa}\right)=\eta_{\kappa}
$$

so that:

$$
p_{\kappa, a_{\kappa}}^{\prime}\left(\varphi_{\kappa}\left(a_{\kappa}\right)\right) \beta_{\kappa}^{\varphi}\left(a_{\kappa}\right) \geq \eta_{\kappa} .
$$

Since $\varphi_{\kappa}\left(a_{\kappa}\right)=0$, the proof of $\Longleftarrow$ follows.

Now, suppose $\varphi_{\kappa}\left(a_{\kappa}\right)>0$. From the first part of Property 1, we deduce:

$$
p_{\kappa, a_{\kappa}}^{\prime}\left(\varphi_{\kappa}\left(a_{\kappa}\right)\right) \beta_{\kappa}^{\varphi}\left(a_{\kappa}\right)=\eta_{\kappa} .
$$

Since $p_{\kappa, a_{\kappa}}^{\prime}$ strictly increases, we obtain:

$$
p_{\kappa, a_{\kappa}}^{\prime}(0) \beta_{\kappa}^{\varphi}\left(a_{\kappa}\right)<\eta_{\kappa}
$$

completing the proof.

Comment Dealing with a discrete (in space) version of this problem, Property 1 follows easily from the Kuhn Tucker theorem. However, the interdependence between the optimality conditions prevents utilization of standard optimization approach based on duality.

\section{Numerical resolution}

\subsection{Algorithm}

We shall develop now an original numerical method for solving our optimization problem. It relies both upon theoretical developments of the previous section and practical considerations. We can split up (15) into the two following equations:

$$
\begin{gathered}
\left(\int_{E} \varphi_{k}(x) d x\right)_{1 \leq k \leq T}=\left(\phi_{j}\right)_{1 \leq k \leq T}, \\
A\left(\phi_{j}\right)_{1 \leq k \leq T}=\left(\psi_{j}\right)_{1 \leq j \leq \Theta} .
\end{gathered}
$$

This gives us a more tractable framework which will be of constant use subsequently.

Now, define the (constraints) vector $\phi \triangleq\left(\phi_{j}\right)_{1 \leq k \leq T}$, as well as the vector 
$\psi \triangleq\left(\psi_{j}\right)_{1<j<\Theta}$. Consider also $A^{\sim}$ a matrix such that $\operatorname{ker} A=\operatorname{Im}\left(A^{\sim}\right)$ and a vector $\phi_{0}$ so that $A \phi_{0}=\psi$. We call $p$ the number of columns of $A^{\sim}$. Then, for each vector $\phi$ fulfilling (34), there is a vector $\nu \in \mathbb{R}^{p}$ so that $\phi=\phi_{0}+\widetilde{A^{\sim} \nu}$. Likewise (34) holds true for all the $\phi$ vectors of the preceding form. The basis of the algorithm is to consider variations of the parameter $\nu$ toward an optimal value for minimizing $\mathbf{P}_{n d}(\varphi)$. For this purpose, we will study the differential behavior of $\min _{\varphi} \mathbf{P}_{n d}(\varphi)$ relatively to $\nu$. Moreover, we will have to take into account the resource positivity constraints.

Two methods have been developed. The first one is more heuristic and uses an approximation hypothesis for computing the differential of $\min _{\varphi} \mathbf{P}_{n d}(\varphi)$ and for choosing the variation $d \nu$. The second one is based upon a deeper analysis and is exact. Remarkably, the results obtained by these two methods are quite similar even if the convergence of the second one is faster.

\subsubsection{An heuristic point of view}

Differential behavior of $\min _{\varphi} \mathbf{P}_{n d}(\varphi)$

Let $\nu \in \mathbb{R}^{p}$ and $\phi=\phi_{0}+\underset{A^{\sim} \nu}{\nu}$. Assume moreover that $\nu$ is such that the resource positivity constraints are satisfied, i.e. $\phi \geq 0$. We then define:

$$
I(\nu) \triangleq\left\{k \in \mathbb{N} / 1 \leq k \leq T \text { and } \phi_{k}>0\right\} .
$$

Let $d \nu$ be a vectorial infinitesimal variation of $\nu$. The infinitesimal variation for $\phi$ is thus $d \phi=A^{\sim} d \nu$. Moreover, let us consider the index subset $J$ and $K$, defined by:

$$
\begin{aligned}
& J(d \nu) \triangleq\left\{k \in \mathbb{N} / 1 \leq k \leq T \text { and } d \phi_{k}>0\right\}, \\
& K(d \nu) \triangleq\left\{k \in \mathbb{N} / 1 \leq k \leq T \text { and } d \phi_{k}<0\right\} .
\end{aligned}
$$

The positivity constraints relative to $\phi$ impose on $d \nu$ the following condition:

Condition $1 \forall k \in\{1, \ldots, T\} \backslash I(\nu), d \phi_{k} \geq 0$.

Let us assume this condition be satisfied and let $\varphi$ be a search distribution minimizing $\mathbf{P}_{n d}$, for the constraint vector $\phi$. Starting from this optimal distribution, we are now dealing with the minimization of $\mathbf{P}_{n d}$ for the resource splitting $\phi+d \phi$ using an elementary point change of $\varphi$. By this way, an approximation of $d \min _{\varphi} \mathbf{P}_{n d}(\varphi)$ will

$\mathrm{RR} \mathrm{n}^{\circ} 3815$ 
be deduced for the vector $\nu$.

Then firstly, we consider a point $\vec{a} \in E^{T}$ and build the following change (denoted $\hat{\varphi}$ ) of $\varphi$ :

$$
\left\{\begin{array}{l}
\forall k \in\{1, \ldots, T\}, \hat{\varphi}_{k}\left(a_{k}\right)=\varphi_{k}\left(a_{k}\right)+d \phi_{k} \\
\hat{\varphi}_{k}\left(x_{k}\right)=\varphi_{k}\left(x_{k}\right) \text { else }
\end{array}\right.
$$

After a first order expansion of $d P=\mathbf{P}_{n d}(\hat{\varphi})-\mathbf{P}_{n d}(\varphi)$ relatively to $d \phi$ and simplification, the following can be written:

$$
d P=\sum_{k=1}^{T} p_{k, a_{k}}^{\prime}\left(\varphi_{k}\left(a_{k}\right)\right) \beta_{k}^{\varphi}\left(a_{k}\right) d \phi_{k} .
$$

To obtain $d \min _{\varphi} \mathbf{P}_{n d}(\varphi)$, we have to choose $\vec{a}$ so that to make $d P$ as negative as possible. The positivity constraint relative to $\hat{\varphi}$ requires that the following condition holds true:

Condition $2 \forall k \in K(d \nu), \varphi_{k}\left(a_{k}\right)>0$.

Then, defining:

$$
X_{k}^{\varphi} \triangleq\left\{x \in E / \varphi_{k}(x)>0\right\},
$$

the following approximation is deduced:

$$
\begin{aligned}
d \min _{\varphi} \mathbf{P}_{n d}(\varphi) \simeq & \sum_{k \in J(d \nu)} \min _{x \in E}\left\{p_{k, x}^{\prime}\left(\varphi_{k}(x)\right) \beta_{k}^{\varphi}(x)\right\} d \phi_{k} \\
& +\sum_{k \in K(d \nu)} \max _{x \in X_{k}^{\varphi}}\left\{p_{k, x}^{\prime}\left(\varphi_{k}(x)\right) \beta_{k}^{\varphi}(x)\right\} d \phi_{k} .
\end{aligned}
$$

\section{Broad lines of the algorithm}

Let $\Delta t$ be a scalar variation (it will be approximated as an infinitesimal). We define $\Delta \nu^{\kappa, \epsilon}$ by $\Delta \nu_{\kappa}^{\kappa, \epsilon}=\epsilon \Delta t$ and $\Delta \nu_{k}^{\kappa, \epsilon}=0$ for $k \neq \kappa$. The algorithm runs in the following way:

1. Compute $A^{\sim}$ and $\phi_{0}$; initialize $\nu, \phi, \varphi$;

2. Choose $\Delta t$ (cf. remark 1$)$; 
3. For each $\kappa \in\{1, \ldots, T\}$ and $\epsilon \in\{-1,+1\}$ define $d \nu=\Delta \nu^{\kappa, \epsilon}$ and compute, when condition (C 1) holds true, the variation $d \min _{\varphi} \mathbf{P}_{n d}(\varphi)$ given by formula (38); select the optimal $d \nu$;

4. Update $\nu$ and $\phi$;

5. For each $\kappa \in\{1, \ldots, T\}$ successively, apply the de Guenin's algorithm to the time-period $\kappa$ in order to optimize $\varphi_{\kappa}$ alone;

6. Return to 2 until convergence.

Remark 1 The choice of $\Delta t$ is in the user's hand. Taking $\Delta t=0$ means convergence step with $\phi$ fixed (it is the Brown's algorithm phase). Taking $\Delta t>0$ signifies gradient descent on $\nu$ (optimizing $\phi$ ). Even if the user has to select it conveniently, this does not induce practical difficulties since the algorithm is quite stable.

\subsubsection{A differential approach}

\section{Differential behavior of $\min _{\varphi} \mathbf{P}_{n d}(\varphi)$}

Assume $\varphi$ be an optimal solution for the vector of resource $\phi$, and assume again that $\varphi+d \varphi$ be an optimal solution for $\phi+d \phi$ ( $d \varphi$ and $d \phi$ are infinitesimal).

The two following equations hold:

$$
\forall k \in\{1, \ldots, T\}, \int_{E} \varphi_{k}(x) d x=\phi_{k}
$$

and

$$
\forall k \in\{1, \ldots, T\}, \int_{E}\left(\varphi_{k}(x)+d \varphi_{k}(x)\right) d x=\phi_{k}+d \phi_{k} .
$$

Thus, we have:

$$
\forall k \in\{1, \ldots, T\}, \int_{E} d \varphi_{k}(x) d x=d \phi_{k} .
$$

In addition, the following optimality condition on $\varphi$ stems from theorem 1 :

$$
\forall k \in\{1, \ldots, T\}, \varphi_{k}(x)>0 \Longrightarrow p_{k, x}^{\prime}\left(\varphi_{k}(x)\right) \beta_{k}^{\varphi}(x)=\eta_{k},
$$

which can be rewritten, using notation (37):

$$
\forall k \in\{1, \ldots, T\}, x \in X_{k}^{\varphi} \Longrightarrow p_{k, x}^{\prime}\left(\varphi_{k}(x)\right) \beta_{k}^{\varphi}(x)=\eta_{k} .
$$


These equations will be instrumental for the final calculation of $d \min _{\varphi} \mathbf{P}_{n d}(\varphi)$.

Now, from the definition $(7), d \min _{\varphi} \mathbf{P}_{n d}(\varphi)$ stands as follows:

$$
\begin{aligned}
d \min _{\varphi} \mathbf{P}_{n d}(\varphi) & =\mathbf{P}_{n d}(\varphi+d \varphi)-\mathbf{P}_{n d}(\varphi)= \\
& \int_{E^{T}} \alpha(\vec{x})\left(\prod_{j=1}^{T}\left(p_{j, x_{j}}\left(\varphi_{j}\left(x_{j}\right)+d \varphi_{j}\left(x_{j}\right)\right) d x_{j}\right)-\prod_{j=1}^{T}\left(p_{j, x_{j}}\left(\varphi_{j}\left(x_{j}\right)\right) d x_{j}\right)\right) .
\end{aligned}
$$

A first order expansion relatively to $d \varphi_{j}\left(x_{j}\right)$ gives the more linear form:

$$
\begin{aligned}
& d \min _{\varphi} \mathbf{P}_{n d}(\varphi)=\sum_{k=1}^{T} \int_{E^{T}} \alpha(\vec{x})\left(\prod_{j \neq k}\left(p_{j, x_{j}}\left(\varphi_{j}\left(x_{j}\right)\right) d x_{j}\right)\right) \\
&\left(p_{k, x_{k}}\left(\varphi_{k}\left(x_{k}\right)+d \varphi_{k}\left(x_{k}\right)\right)-p_{k, x_{k}}\left(\varphi_{k}\left(x_{k}\right)\right)\right) d x_{k}
\end{aligned}
$$

which can be rewritten as:

$$
d \min _{\varphi} \mathbf{P}_{n d}(\varphi)=\sum_{k=1}^{T} \int_{E} \beta_{k}^{\varphi}(x)\left(p_{k, x}\left(\varphi_{k}(x)+d \varphi_{k}(x)\right)-p_{k, x}\left(\varphi_{k}(x)\right)\right) d x .
$$

Since minimization of a sum reverts to minimizing each member of this sum, our initial problem reduces to optimizing, for each index $\kappa$, the variation $d \varphi_{\kappa}$ so as to minimize the following 1-dimensional integrals :

$$
\int_{E} \beta_{\kappa}^{\varphi}(x) p_{\kappa, x}\left(\varphi_{\kappa}(x)+d \varphi_{\kappa}(x)\right) d x
$$

The above expression is minimized under the constraint (40) and it occurs even when each $\varphi_{k}$ is maintained constant. Assuming all $\varphi_{k}$ constant means that $\beta_{\kappa}^{\varphi}$ is constant too. Then the optimization of $d \varphi_{\kappa}$, which is equivalent to the optimization of $\varphi_{\kappa}+d \varphi_{\kappa}$ in (47), reduces to a 1-period search problem of de Guenin. This problem is clearly solved by means of the de Guenin optimality conditions :

$$
\left(\varphi_{\kappa}+d \varphi_{\kappa}\right)(x)>0 \Longrightarrow p_{\kappa, x}^{\prime}\left(\varphi_{\kappa}(x)+d \varphi_{\kappa}(x)\right) \beta_{\kappa}^{\varphi}(x)=c_{\kappa},
$$

which can be rewritten, using notation (37) and defining $c_{\kappa}=\eta_{\kappa}+d \eta_{\kappa}$ :

$$
x \in X_{\kappa}^{\varphi+d \varphi} \Longrightarrow p_{\kappa, x}^{\prime}\left(\varphi_{\kappa}(x)+d \varphi_{\kappa}(x)\right) \beta_{\kappa}^{\varphi}(x)=\eta_{\kappa}+d \eta_{\kappa} .
$$

We then must consider two cases. 
First case: $\phi_{\kappa}>0$

Using notation $W_{\kappa}^{\varphi} \triangleq X_{\kappa}^{\varphi+d \varphi} \cap X_{\kappa}^{\varphi}$ and $Y_{\kappa}^{\varphi} \triangleq X_{\kappa}^{\varphi+d \varphi} \cup X_{\kappa}^{\varphi}$, subtraction of (43) from (49) gives:

$$
\begin{aligned}
& \forall x \in W_{\kappa}, \beta_{\kappa}^{\varphi}(x) p_{\kappa, x}^{\prime \prime}\left(\varphi_{\kappa}(x)\right) d \varphi_{\kappa}(x)=d \eta_{\kappa} \\
& \forall x \in E \backslash Y_{\kappa}, d \varphi_{\kappa}(x)=0
\end{aligned}
$$

Since we consider integration on the frontier $Y_{\kappa}^{\varphi} \backslash W_{\kappa}^{\varphi}$, the values $\int_{Y_{\kappa}^{\varphi} \backslash W_{\kappa}^{\varphi}} \varphi_{\kappa}(x) d x$ and $\int_{Y_{\kappa}^{\varphi} \backslash W_{\kappa}^{\varphi}}\left(\varphi_{\kappa}(x)+d \varphi_{\kappa}(x)\right) d x$ are second order infinitesimals (an easy proof left to the reader). The following equality then holds:

$$
\int_{X_{\kappa}^{\varphi}} \frac{d \eta_{\kappa} d x}{\beta_{\kappa}^{\varphi}(x) p_{\kappa, x}^{\prime \prime}\left(\varphi_{\kappa}(x)\right)}=\int_{X_{\kappa}^{\varphi}} d \varphi_{\kappa}(x) d x=\int_{E} d \varphi_{\kappa}(x) d x=d \phi_{\kappa},
$$

so that:

$$
d \eta_{\kappa}=d \phi_{\kappa} / \int_{X_{\kappa}^{\varphi}} \frac{d x}{\beta_{\kappa}^{\varphi}(x) p_{\kappa, x}^{\prime \prime}\left(\varphi_{\kappa}(x)\right)}
$$

and, finally :

$$
\forall x \in W_{\kappa}^{\varphi}, d \varphi_{\kappa}(x)=\frac{d}{\phi_{\kappa}} \beta_{\kappa}^{\varphi}(x) p_{\kappa, x}^{\prime \prime}\left(\varphi_{\kappa}(x)\right) / \int_{X_{\kappa}^{\varphi}} \frac{d x}{\beta_{\kappa}^{\varphi}(x) p_{\kappa, x}^{\prime \prime}\left(\varphi_{\kappa}(x)\right)} .
$$

Then, the $\kappa^{t h}$ component of $d \min _{\varphi} \mathbf{P}_{n d}(\varphi)$ is derived as follows:

$$
\begin{aligned}
\int_{E} \beta_{\kappa}^{\varphi}(x)\left(p_{\kappa, x}\left(\varphi_{\kappa}(x)+d \varphi_{\kappa}(x)\right)\right. & \left.-p_{\kappa, x}\left(\varphi_{\kappa}(x)\right)\right) d x= \\
& =\int_{E} \beta_{\kappa}^{\varphi}(x) p_{\kappa, x}^{\prime}\left(\varphi_{\kappa}(x)\right) d \varphi_{\kappa}(x) d x \\
& =\int_{X_{\kappa}^{\varphi}} \beta_{\kappa}^{\varphi}(x) p_{\kappa, x}^{\prime}\left(\varphi_{\kappa}(x)\right) d \varphi_{\kappa}(x) d x \\
& =\int_{X_{\kappa}^{\varphi}} \frac{p_{\kappa, x}^{\prime}\left(\varphi_{\kappa}(x)\right)}{p_{\kappa, x}^{\prime \prime}\left(\varphi_{\kappa}(x)\right)} d x / \int_{X_{\kappa}^{\varphi}} \frac{d x}{\beta_{\kappa}^{\varphi}(x) p_{\kappa, x}^{\prime \prime}\left(\varphi_{\kappa}(x)\right)} d \phi_{\kappa}
\end{aligned}
$$

Second case: $\phi_{\kappa}=0$

Numerous problems stem from the nullity of $\phi_{\kappa}$. First, we could remark that $\varphi_{\kappa}=0$, so that equation (43) makes no sense although it is exact. Another difficulty is that 
we must restrict to non negative variations of $\phi_{\kappa}$ and $\varphi_{\kappa}$. The variation of $\varphi_{\kappa}$, associated with the variation $d \phi_{\kappa}$ of $\phi_{\kappa}$, will be denoted $\delta \varphi_{\kappa}$ rather than $d \varphi_{\kappa}$ (in this case it is not sure that the variations of $\phi_{\kappa}$ and $\varphi_{\kappa}$ are of the same order of magnitude). Moreover, $\delta \eta_{\kappa}$ will denote the variation of $\eta_{\kappa}$. It is then easy to show that $\delta \varphi_{\kappa}$ and $\delta \eta_{\kappa}$ are same order infinitesimals. These variations will be related to the maximum value $\eta_{\kappa}^{0}=\max _{x \in E}\left(\beta_{\kappa}^{\varphi}(x) p_{\kappa, x}^{\prime}(0)\right)$. We have the following coming from de Guenin's equation:

$$
\beta_{\kappa}^{\varphi}(x) \leq \frac{\eta_{\kappa}^{0}+\delta \eta_{\kappa}}{p_{\kappa, x}^{\prime}(0)} \Longrightarrow \delta \varphi_{\kappa}(x)=0
$$

We deduce that $\beta_{\kappa}^{\varphi}(x) p_{\kappa, x}^{\prime}(0)=\eta_{\kappa}^{0}$ with an infinitesimal approximation, when $\delta \varphi_{\kappa}(x)>0$. A simple expression of the $\kappa^{t h}$ component of $d \min _{\varphi} \mathbf{P}_{n d}(\varphi)$ is then:

$$
\begin{gathered}
\int_{E} \beta_{\kappa}^{\varphi}(x)\left(p_{\kappa, x}\left(\varphi_{\kappa}(x)+\delta \varphi_{\kappa}(x)\right)-p_{\kappa, x}\left(\varphi_{\kappa}(x)\right)\right) d x=\int_{E} \beta_{\kappa}^{\varphi}(x) p_{\kappa, x}^{\prime}(0) \delta \varphi_{\kappa}(x) d x \\
=\int_{\beta_{\kappa}^{\varphi}(x) \geq \frac{\eta_{\kappa}^{0}+\delta \eta_{\kappa}}{p_{\kappa, x}^{\prime}(0)}} \beta_{\kappa}^{\varphi}(x) p_{\kappa, x}^{\prime}(0) \delta \varphi_{\kappa}(x) d x \\
=\int_{\beta_{\kappa}^{\varphi}(x) \geq \frac{\eta_{\kappa}^{0}+\delta \eta_{\kappa}}{p_{\kappa, x}^{\prime}(0)}} \eta_{\kappa}^{0} \delta \varphi_{\kappa}(x) d x \\
=\eta_{\kappa}^{0} \int_{E} \delta \varphi_{\kappa}(x) d x=\eta_{\kappa}^{0} d \phi_{\kappa} .
\end{gathered}
$$

\section{Calculation of $d \min _{\varphi} \mathbf{P}_{n d}(\varphi)$}

Let us define the vector $\mathbf{V}$ with $T$ components by:

$$
\left\{\begin{array}{c}
\mathbf{V}_{k}=\frac{\int_{X_{k}^{\varphi}} \frac{p_{k, x}^{\prime}\left(\varphi_{k}(x)\right)}{p_{k, x}^{\prime \prime}\left(\varphi_{k}(x)\right)} d x}{\int_{X_{k}^{\varphi}} \frac{d x}{\beta_{k}^{\varphi}(x) p_{k, x}^{\prime \prime}\left(\varphi_{k}(x)\right)}} \text { when } \phi_{k}>0 \\
\mathbf{V}_{k}=\eta_{k}^{0} \text { when } \phi_{k}=0 .
\end{array}\right.
$$


Thus, $d \min _{\varphi} \mathbf{P}_{n d}(\varphi)$ may be rewritten as:

$$
\begin{aligned}
d \min _{\varphi} \mathbf{P}_{n d}(\varphi) & =\sum_{k} \int_{E} \beta_{k}^{\varphi}(x)\left(p_{k, x}\left(\varphi_{k}(x)+d \varphi_{k}(x)\right)-p_{k, x}\left(\varphi_{k}(x)\right)\right) d x \\
& =\mathbf{V}^{t} d \phi .
\end{aligned}
$$

Now, let us consider $\nu \in \mathbb{R}^{p}$ and $\phi=\phi_{0}+A^{\sim} \nu$. Suppose moreover that the resource positivity constraints are satisfied for $\nu$. Let $d \nu$ be a vectorial infinitesimal variation of $\nu$ so that resource positivity constraints for $\nu+d \nu$ still hold. The infinitesimal variation for $\phi$ is thus $d \phi=A^{\sim} d \nu$, and we deduce:

$$
d \min _{\varphi} \mathbf{P}_{n d}(\varphi)=\mathbf{V}^{t} A^{\sim} d \nu
$$

\section{Computing the optimal variation $\Delta \nu$}

Practically, our goal is to compute an optimal variation $\Delta \nu$ of $\nu$ for optimizing the variation of the probability of non detection. The choice of $\Delta \nu$ is limited by a maximal value (say $\Delta t$ ) of the norm of $\Delta \phi=A^{\sim} \Delta \nu$. We will treat $\Delta \nu$ as an infinitesimal, so as to use result (59). This leads us to consider the following optimization problem:

Property 2 The optimal variation $\Delta \nu$ of $\nu \in \operatorname{ker} B$ (subject to $\|\Delta \phi\| \leq \Delta t$ ) is the result of the following optimization:

$$
\begin{array}{ll}
\text { Minimize } & \mathbf{V}^{t} A^{\sim} \Delta \nu, \\
\text { subject to } & \\
& A^{\sim} \Delta \nu+\phi \geq 0, \\
& \left\|A^{\sim} \Delta \nu\right\| \leq \Delta t .
\end{array}
$$

When \|\|$=\|\|_{\infty}$, that is $\|\phi\|=\sup _{k}\left|\phi_{k}\right|$, the problem is linear and can be straightforwardly solved by means of the Simplex algorithm.

\section{Broad lines of the algorithm}

Let $\Delta t$ be a scalar variation. The algorithm runs in the following way:

1. Compute $A^{\sim}$ and $\phi_{0}$; initialize $\nu, \phi, \varphi$;

2. Choose $\Delta t$ (cf. remark 1$)$;

3. Compute $\Delta \nu$ by optimizing (60); 
4. Update $\nu$ and $\phi$;

5. For each $\kappa \in\{1, \ldots, T\}$ successively, apply the de Guenin's algorithm to the time-period $\kappa$ in order to optimize $\varphi_{\kappa}$ alone;

6. Return to 2 until convergence.

\subsubsection{A la Brown implementation}

Practically, a problem we have to face with this algorithm stems from the fact that the computation of the function $\beta_{\kappa}^{\varphi}$ requires a huge amount of computation time. Calculating this function needs integration on the $(T-1)$-dimensional space

$E^{T-1}$, for each element of $E$. If we consider as a time unit $u$ integration on $E$, the computation time of $\beta_{\kappa}^{\varphi}$ is of an $u^{T}$ order. Finally, the computation of steps 2 and 5 requires about $2 T u^{T}$. Practically, the parameter $u$ is rather large which means that this (direct) approach is clearly infeasible.

In order to overcome this difficulty, the idea of Brown will be again instrumental. More precisely, we shall see that the Markovian property of $\alpha$ can drastically reduce the computation requirements. Let us now detail this approach. For $x \in E$, let us define $U^{\varphi}(x)$ and $D^{\varphi}(x)$ in the following recursive way:

$$
\begin{aligned}
& U_{1}^{\varphi}(x)=1 \text { and } D_{T}^{\varphi}(x)=1, \\
& U_{k+1}^{\varphi}(x)=\int_{E} \alpha_{k, k+1}(y, x) p_{k, y}\left(\varphi_{k}(y)\right) U_{k}^{\varphi}(y) d y, \\
& D_{k-1}^{\varphi}(x)=\int_{E} \alpha_{k-1, k}(x, y) p_{k, y}\left(\varphi_{k}(y)\right) D_{k}^{\varphi}(y) d y .
\end{aligned}
$$

Computing $U_{k}^{\varphi}$ knowing $U_{k-1}^{\varphi}$ or computing $D_{k}^{\varphi}$ knowing $D_{k+1}^{\varphi}$ requires a time of the order of $u^{2}$. Altogether, computing all $U^{\varphi}$ and $D^{\varphi}$ require $2 T u^{2}$. Assuming $U^{\varphi}$ and $D^{\varphi}$ available, then we compute $\beta^{\varphi}$ as a simple product:

$$
\beta_{k}^{\varphi}(x)=U_{k}^{\varphi}(x) D_{k}^{\varphi}(x) .
$$

A refinement allows to spar even more computation time. Usually, since $\varphi$ is changed, we have to compute $U^{\varphi}$ and $D^{\varphi}$ again. But we can remark that, when only $\varphi_{\kappa}$ is changed, $U_{k}^{\varphi}$ and $D_{l}^{\varphi}$ stay unchanged for $k \leq \kappa$ and $l \geq \kappa$.

Finally, our algorithm then takes the following form: 
1. Compute $A^{\sim}, \phi_{0}$; initialize $\nu, \phi, \varphi$; compute $U^{\varphi}$;

2. Compute $D^{\varphi}$;

3. Choose $\Delta t$ (cf. remark 1);

4. Find $\Delta \nu$ optimizing $d \min _{\varphi} \mathbf{P}_{n d}(\varphi)$;

5. Update $\nu$ and $\phi$;

6. For $\kappa$ going from 1 to $T$, compute:

- Compute $U_{\kappa}^{\varphi}$;

- Compute $\varphi_{\kappa}$ by the de Guenin's algorithm;

7. Go back to 2 until convergence.

The time for computing a complete iteration is of the order of $2 T u^{2}$. The algorithm then computes the optimal $\Delta \nu$ and runs the de Guenin's algorithm one time for each time-period of search. Remark that if we suppress stages 3,4 and 5 out of the program, or if we force $\Delta t=0$, we go back roughly to the F.A.B. algorithm.

\section{$5 \quad$ Extension to mixed resources}

\subsection{Examples}

\subsubsection{Mixing resources with different features}

Let us consider a search for a target on a space $E$ involving three types of resource; e.g. non renewable resources (denoted $a$ ), 2-period renewable resources (denoted $b$ ) and 3-period renewable resources (denoted $c$ ). Assume that the search duration is $T$ (periods). We call $N_{a}, N_{b}$, and $N_{c}$ the amounts of available resource for each type $a, b$, and $c$. In the same way $\varphi^{a}, \varphi^{b}$ and $\varphi^{c}$ represent the function of (local) search effort for the types $a, b$, and $c$. The renew hypothesis on $a, b$ and $c$ leads to consider the three following types of constraints:

$$
A^{a}\left(\int_{E} \varphi_{k}^{a}(x) d x\right)_{1 \leq k \leq T}=\psi^{a}, \text { where } A^{a}=A_{N R}=(1 \ldots 1) \text { and } \psi^{a}=\left(N_{a}\right)
$$




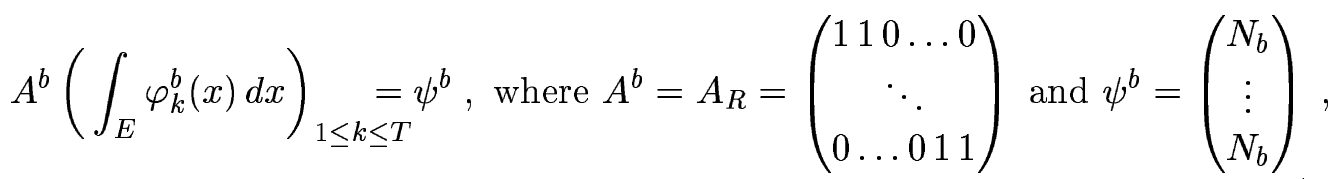

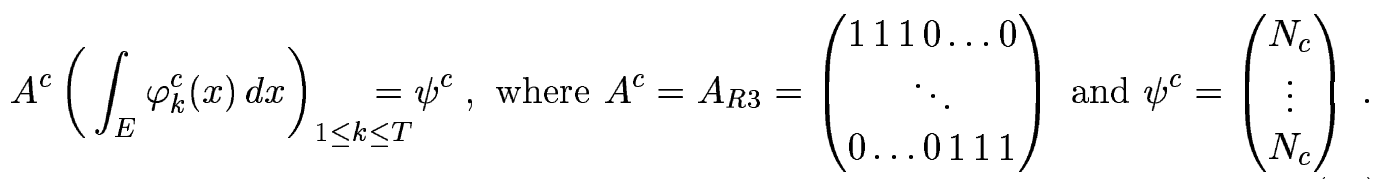

Let us define:

$$
A^{a b c}=\left(\begin{array}{ccc}
A^{a} & 0 & 0 \\
0 & A^{b} & 0 \\
0 & 0 & A^{c}
\end{array}\right) \text { and } \psi^{a b c}=\left(\begin{array}{c}
\psi^{a} \\
\psi^{b} \\
\psi^{c}
\end{array}\right)
$$

constraints (63), (64) and (65) then take the following (concatenated) form:

$$
A^{a b c}\left(\begin{array}{c}
\left.\int_{E} \varphi_{k}^{a}(x) d x\right|_{1 \leq k \leq T} \\
\left.\int_{E} \varphi_{k}^{b}(x) d x\right|_{1 \leq k \leq T} \\
\left.\int_{E} \varphi_{k}^{c}(x) d x\right|_{1 \leq k \leq T}
\end{array}\right)=\psi^{a b c}
$$

Moreover, assuming the independence of elementary non-detection functions, the probability of non detection stands as follows:

$\mathbf{P}_{n d}\left(\varphi^{a}, \varphi^{b}, \varphi^{c}\right)=\int_{E^{T}} \alpha(\vec{x}) \prod_{k=1}^{k=T}\left(p_{k, x_{k}}^{a}\left(\varphi_{k}^{a}\left(x_{k}\right)\right) p_{k, x_{k}}^{b}\left(\varphi_{k}^{b}\left(x_{k}\right)\right) p_{k, x_{k}}^{c}\left(\varphi_{k}^{c}\left(x_{k}\right)\right)\right) \prod_{k=1}^{k=T} d x_{k}$,

where $p^{a}, p^{b}$ and $p^{c}$ are functions specific to $a, b$ and $c$.

To optimize the search, we have then to minimize $\mathbf{P}_{n d}\left(\varphi^{a}, \varphi^{b}, \varphi^{c}\right)$ under the constraint (67) and the positivity of $\varphi^{a}, \varphi^{b}, \varphi^{c}$.

\subsubsection{Resources with different operating modes}

Assume that we dispose of a single type of resource with matrix and vector constraints $A$ and $\psi$, but that those resources can run in several different ways. We will call 
those different ways the operating modes. Such problem is still simply modeled as previously. For an example, assume that our search resource can operate in two different modes $a$ and $b$. We call $\varphi^{a}$ and $p^{a}$ the functions for mode $a$ and $\varphi^{b}$ and $p^{b}$ the functions for mode $b$. Then, describing the sharing of the resources between mode $a$ and mode $b$, the following relation holds:

$$
A\left(\int_{E} \varphi_{k}^{a}(x) d x+\int_{E} \varphi_{k}^{b}(x) d x\right)_{1 \leq k \leq T}=\psi
$$

The resources are thus period-shared following the constraint $(A, \psi)$ and, as this period-sharing is done, the resource's operating mode has to be chosen by splitting between mode $a$ and mode $b$ during the period. In addition, the probability of non detection is rewritten:

$$
\mathbf{P}_{n d}\left(\varphi^{a}, \varphi^{b}\right)=\int_{E^{T}} \alpha(\vec{x}) \prod_{k=1}^{k=T}\left(p_{k, x_{k}}^{a}\left(\varphi_{k}^{a}\left(x_{k}\right)\right) p_{k, x_{k}}^{b}\left(\varphi_{k}^{b}\left(x_{k}\right)\right)\right) \prod_{k=1}^{k=T} d x_{k} .
$$

Let us define:

$$
A^{a b}=(A A)
$$

The condition (69) becomes:

$$
A^{a b}\left(\begin{array}{c}
\left.\int_{E} \varphi_{k}^{a}(x) d x\right|_{1 \leq k \leq T} \\
\left.\int_{E} \varphi_{k}^{b}(x) d x\right|_{1 \leq k \leq T}
\end{array}\right)=\psi
$$

To optimize the search, we have then to minimize $\mathbf{P}_{n d}\left(\varphi^{a}, \varphi^{b}\right)$ under the constraint (72) and the positivity of $\varphi^{a}, \varphi^{b}$.

\subsection{General formulation}

In fact, all these problems can be immersed in a common formalism. A target moving in space $E$ is to be searched. The search being dispatched in $T$ periods, we denote $\alpha(\vec{x})$ the density probability of the target trajectory. The resources used for the search are of $r$ different types, associated with the non detection functions $\left(p^{\rho}\right)_{1 \leq \rho \leq r}$. We denote $\varphi=\left(\varphi_{k}^{\rho}\right)_{1 \leq k \leq T ; 1 \leq \rho \leq r}$ the corresponding (local) effort functions. Assuming the hypothesis of independence of searches, the following value of 
the non detection probability is obtained:

$$
\mathbf{P}_{n d}(\boldsymbol{\varphi})=\int_{E^{T}} \alpha(\vec{x}) \prod_{k=1}^{T}\left(\left(\prod_{\rho=1}^{r} p_{k, x_{k}}^{\rho}\left(\varphi_{k}^{\rho}\left(x_{k}\right)\right)\right) d x_{k}\right)
$$

As seen previously, we have to consider the following constraint:

$$
\boldsymbol{A}\left(\left.\int_{E} \varphi_{k}^{\rho}(x) d x\right|_{1 \leq k \leq T}\right)_{1 \leq \rho \leq r}=\Psi
$$

where $\boldsymbol{A}$ is a matrix and $\Psi$ is a vector such that $\boldsymbol{A} X=\Psi$ admits a solution.

Our aim is then to optimize $\varphi$ in order to minimize $\mathbf{P}_{n d}(\varphi)$, defined by equation (73), under the constraints (74) and positivity of $\varphi$. The general algorithm for solving this optimization problem is presented in Appendix A.

\section{Inequality constraints}

\subsection{Inequalities are best}

So far, only equality constraints have been considered (see e.g. section 3 ). In fact, the equality constraints (15) are somewhat restrictive; especially in cases where it may be profitable to keep some resource unused so that to use them more efficiently in later period. For this reason, it could be of interest to optimize under inequality constraints. The problem setting is changed this way:

$$
\begin{aligned}
& \text { Minimize } \mathbf{P}_{n d}(\varphi)=\int_{E^{T}} \alpha(\vec{x}) \prod_{k=1}^{k=T} p_{k, x_{k}}\left(\varphi_{k}\left(x_{k}\right)\right) \prod_{k=1}^{k=T} d x_{k} \\
& \text { subject to } A\left(\int_{E} \varphi_{k}(x) d x\right)_{1 \leq k \leq T} \leq \psi
\end{aligned}
$$


By considering the following (split) formulation:

$$
\begin{aligned}
& \text { Minimize } \mathbf{P}_{n d}(\varphi)=\int_{E^{T}} \alpha(\vec{x}) \prod_{k=1}^{k=T} p_{k, x_{k}}\left(\varphi_{k}\left(x_{k}\right)\right) \prod_{k=1}^{k=T} d x_{k} \\
& \text { subject to } A \phi \leq \psi \\
& \text { and }\left(\int_{E} \varphi_{k}(x) d x\right)_{1 \leq k \leq T}=\phi
\end{aligned}
$$

the problem stands in our general optimization framework (see nest section).

\subsection{Algorithm}

In fact, we will translate the inequality constraints into equality ones by means of slack variables. More precisely, let us denote $\Theta$ the row number of the (constraint) matrix $A$, then inequality constraints $A \phi \leq \psi$ revert to consider equality constraints ( $A \phi+\phi^{p}=\psi$ ) by adding $\Theta$ slack variables $\phi_{1}^{p}, \ldots, \phi_{\Theta}^{p}$ (satisfying also to the positivity constraints $\left.\phi_{k}^{p} \geq 0\right)$ to each row of $A \phi=\psi$. Denoting $I^{\Theta}$ the $\Theta$-dimensional identity matrix, the preceding optimization problem then becomes (details in appendix B):

$$
\begin{aligned}
& \text { Inequality constraints } \longrightarrow \text { Equality constraints } \\
& A \phi \leq \psi \quad \longrightarrow \quad\left(A I_{\Theta}\right)\left(\begin{array}{c}
\phi \\
\phi^{p}
\end{array}\right)=\psi \\
& \phi \geq 0 \quad \longrightarrow \quad\left(\begin{array}{c}
\phi \\
\phi^{p}
\end{array}\right) \geq 0 \\
& \mathbf{P}_{n d}(\varphi)=\int_{E^{T}} \alpha(\vec{x}) \prod_{k=1}^{k=T} p_{k, x_{k}}\left(\varphi_{k}\left(x_{k}\right)\right) \prod_{k=1}^{k=T} d x_{k} \text { and } \\
& \left(\int_{E} \varphi_{k}(x) d x\right)_{1 \leq k \leq T}=\phi \text { stay unchanged }
\end{aligned}
$$

Roughly the algorithm is unchanged, except the $A$ matrix which is replaced by $\left(A I_{\Theta}\right)$. Nevertheless matrix $\left(A I_{\Theta}\right)$ is used since $A$. However we stress that, since $\varphi^{p}$ has no physical meaning (slack variables), the components of $\mathbf{V}$ associated with the variables $\phi^{p}$ are zeroed (calculation of an optimal $\Delta \nu$ ).

$\mathrm{RR} \mathrm{n}^{\circ} 3815$ 


\section{Results}

The space search $E$ is a square of $30 \times 30$ cells. Target's trajectories are simulated within the following general scheme: a start position, a motion componant and a possibly final position.

The target starting position is represented by an uniform density in the $10 \times 10$ square, centered around the point $(5,5)$, i.e. :

$$
\begin{aligned}
& s(x)=\frac{1}{100} \text { if }(5,5) \leq x \leq(14,14) \\
& s(x)=0 \text { else. }
\end{aligned}
$$

The density of the (possibly) final target location is uniform in the $10 \times 10$ square (center: $(16,16)$ and is denoted $f$ :

$$
\begin{aligned}
& f(x)=\frac{1}{100} \text { if }(16,16) \leq x \leq(25,25) \\
& f(x)=0 \text { else. }
\end{aligned}
$$

At each time-period the (Markovian) target motion is an uniform diffusion (toward down and right) represented by the function $m$ :

$$
\left\{\begin{array}{l}
m(0,0)=m(3,3)=\frac{3}{14} \text { et } m(2,3)=m(3,2)=\frac{2}{14} \\
m(0,3)=m(3,0)=m(1,3)=m(3,1)=\frac{1}{14} \\
m(\mathbf{x})=0, \text { else }
\end{array}\right.
$$

For example, the density $(\alpha(\vec{x}))$ of a target trajectory (e.g. for 4 time-period scenario) could take the following form:

$$
\alpha(\vec{x})=Z \times s\left(x_{1}\right) m\left(x_{2}-x_{1}\right) m\left(x_{3}-x_{2}\right) m\left(x_{4}-x_{3}\right) f\left(x_{4}\right) .
$$

It represents a down-right diffusion movement diverging at the beginning from the starting square and finally converging back to the final square. The value $Z$ represents a normalization term.

The test results are divided into three sections. In the first one, we shall examine the effects of the form of non detection functions $p$ (only uniform functions over $x$ will be considered). In the second one, examples with mixed resources or multiple modes will be presented ( $p_{x}$ non uniform). Finally, inequality constraints are examined in the last one. 


\subsection{Effects of the detection function $p$}

Throughout this section we shall consider a unique modeling of the target motion and a 4 time-period search. The target distribution function is:

$$
\alpha(\vec{x})=s\left(x_{1}\right) m\left(x_{2}-x_{1}\right) m\left(x_{3}-x_{2}\right) m\left(x_{4}-x_{3}\right)
$$

Only one type of resource is used. The non detection functions of these resources are uniform, that is:

$$
\forall k \forall x \in E, p_{k, x}=\pi,
$$

where $\pi$ is a common non detection function. We shall compare results obtained by an exponential (non-detection) function (i.e. $\pi(\varphi)=\exp (\omega \varphi) ; \omega=1$ ) on the first hand and a non exponential one (i.e. $\left.\pi(\varphi)=1 /(\varphi+1)^{2}\right)$ on the second. Exponential functions are widely used even if restricting assumptions are underlined (detection without loss).

\subsubsection{Exponential function}

Under this assumption, the whole effort can be entirely affected to a unique timeperiod without any loss. The essential of search effort is then brutally put on the periods where the target density is the more concentrated. This behavior is illustrated by the following example. Here, resources are not renewable ( $A=A_{N R}$ and $\psi=\psi_{N R}$ see section 3.1.1), and the total effort is $N=20$. The splitting of search efforts between the consecutive periods is illustrated by Table 1, while the spatiotemporal evolution of the distribution of search efforts is visualized by sequence of one-period figures (Figure 2). We can see that the significant search resources (dark

\begin{tabular}{|c|c|c|c|c|c|c|}
\hline$A$ & $N$ & $\phi_{1}$ & $\phi_{2}$ & $\phi_{3}$ & $\phi_{4}$ & Figure \\
\hline \hline$A_{N R}$ & 20 & 20 & 0 & 0 & 0 & 2 \\
\hline
\end{tabular}

Table 1: Exponential function

areas) are strongly concentrated on the areas with the higher probability of presence of the target, that is on the first period for this diffusive target. No search is done on the three last periods. This result is obtained for any other values of $N$.

\subsubsection{Non exponential function}

In such case, the whole effort cannot be entirely affected at the same time-period without significant loss. We present three results. The first one using $A=A_{N R}$ and 


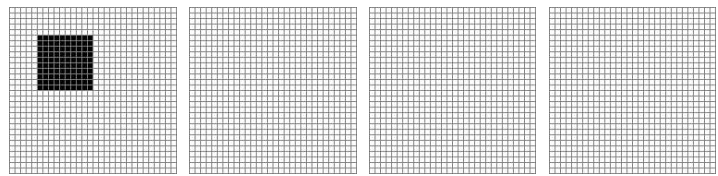

Figure 2: Non renewable resources; $A=A_{N R}, N=20$; exponential function.

$\psi=\psi_{N R}$, where $N=200$. The two other ones using $A=A_{R}$ and $\psi=\psi_{R}$, with $N=100$, respectively $N=10$.

\begin{tabular}{|c|c|c|c|c|c|c|c|}
\hline$A$ & $N$ & $\phi_{1}$ & $\phi_{2}$ & $\phi_{3}$ & $\phi_{4}$ & $\mathbf{P}_{n d}(\varphi)$ & Figure \\
\hline \hline$A_{N R}$ & 200 & 112 & 50 & 25 & 13 & $7,7 \%$ & 3 \\
\hline$A_{R}$ & 100 & 73 & 27 & 73 & 27 & $9,1 \%$ & 4 \\
\hline$A_{R}$ & 10 & 8,7 & 1,3 & 8,7 & 1,3 & $71 \%$ & 5 \\
\hline
\end{tabular}

Table 2: Non exponential function

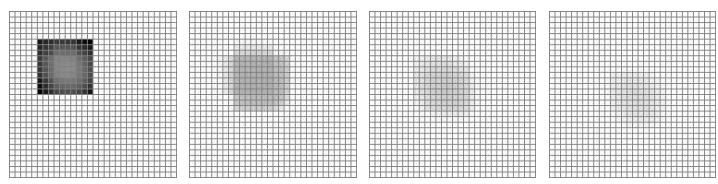

Figure 3: Non renewable resources; $A=A_{N R}, N=200$; Non exponential function.

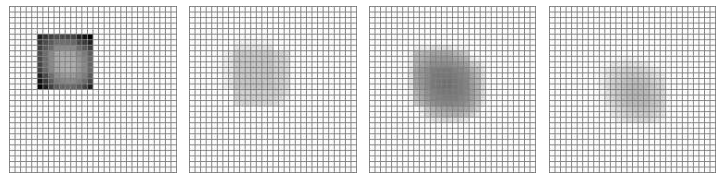

Figure 4: Renewable resources; $A=A_{R}, N=100$; Non exponential function.

Table 2 illustrates, for the three scenarios, the splitting of search effort at each timeperiod $\left(\phi_{1}, \phi_{2}, \phi_{3}, \phi_{4}\right)$. Moreover the optimal probability $\mathbf{P}_{n d}$ is given, since the results are here comparable. The significant resources are still concentrated on the areas with the higher probability of presence of the target. But the period-splitting 


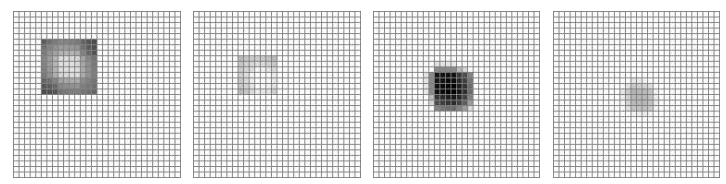

Figure 5: Renewable resources; $A=A_{R}, N=10$; Non exponential function.

is smoother, although the effort still tends to be concentrated on the first timeperiods. The splitting is more rough (low resources make loss decreasing) when the total resources are small (second and third example: $\phi_{1}$ and $\phi_{3}$ increase from $73 \%$ of resources to $87 \%$ ). Nevertheless, we notice a surrounding strategy for the distribution of the search effort on the first periods (figures 3,4 and especially 5 ).

\subsection{Mixing of resources or modes}

From now on, all the non detection functions will be exponential. In this section, we present examples with mixed search resources. Moreover, the non detection functions will be depending on the space location and on the type $\rho$ of search resource (but independent of $k$ ), so that $p_{k, x}^{\rho}(\varphi)=\exp \left(\omega_{x}^{\rho} \varphi\right)$. For each example, the search duration is 7 periods. The target distribution corresponds to a diffusion from the start position:

$$
\alpha(\vec{x})=s\left(x_{1}\right) \prod_{k=1}^{6} m\left(x_{k+1}-x_{k}\right)
$$

\subsubsection{Multiple resources}

A simple example of mixed resources (such as in section 5.1.1) is presented. Two different resources are used. The first resource $(\rho=1)$ is a non renewable one with constraint matrix $A=A_{N R}$ and total resource $N=100$. The second resource $(\rho=2)$ is a 3 periods-renewable resource with constraint matrix $A=A_{R 3}$ (see section 5.1.1) and total resource $N=50$. The exponential parameter of first resource, say $\omega_{x}^{1}$, is decreasing from the right-down bottom of the search space $E$. The variation of $\omega_{x}^{1}$ is more detailed in the $8^{\text {th }}$ (from left) picture of figure 6 . The exponential parameter of second resource, say $\omega_{x}^{2}$, is decreasing from the left-down bottom of the search space $E$ and is detailed in the $8^{\text {th }}$ picture of figure 7 .

The optimal splitting of the two resources is presented in table 3. Figure 6 represents the spatial sharing of the first resource for each seven periods (see the first seven 


\begin{tabular}{|c|c|c|c|c|c|c|c|c|c|c|}
\hline$A$ & $N$ & $\rho$ & $\phi_{1}^{\rho}$ & $\phi_{2}^{\rho}$ & $\phi_{3}^{\rho}$ & $\phi_{4}^{\rho}$ & $\phi_{5}^{\rho}$ & $\phi_{6}^{\rho}$ & $\phi_{7}^{\rho}$ & Figure \\
\hline \hline$A_{N R}$ & 100 & 1 & 82 & 2.8 & 0.2 & 0 & 0 & 0 & 15 & 6 \\
\hline$A_{R 3}$ & 50 & 2 & 50 & 0 & 0 & 50 & 0 & 0 & 50 & 7 \\
\hline
\end{tabular}

Table 3: Mixed resources.
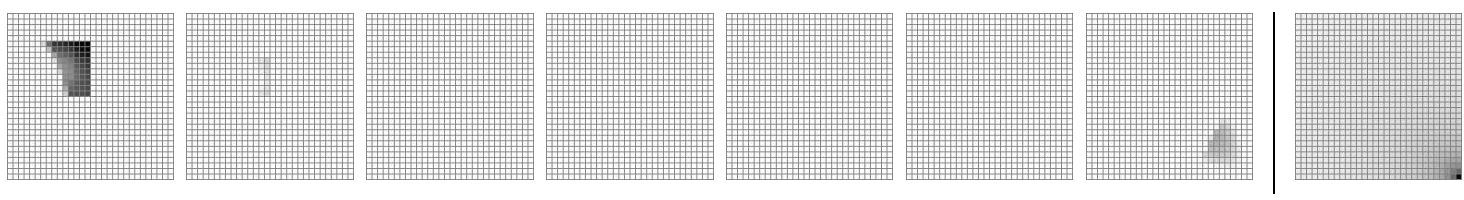

Figure 6: Type 1; non renewable resources; $A=A_{N R}, N=100$.
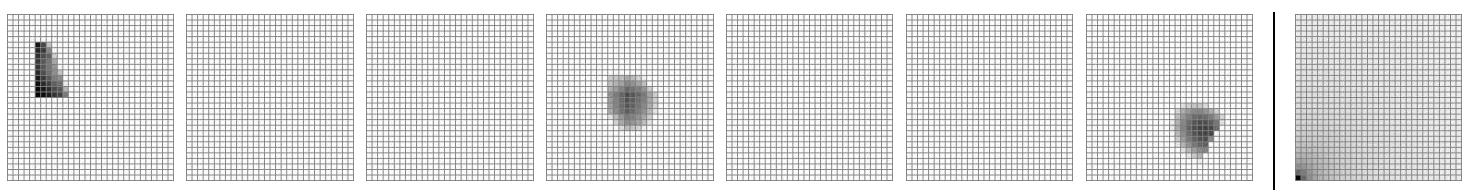

Figure 7: Type 2; renewable resources; $A=A_{R 3}, N=50$.

pictures). Figure 7 represents the spatial sharing of the second resource. The second resource splitting is $50,0,0,50,0,0,50$. This result appears quite natural since it gives the higher amount of resource. Moreover, the target spread tends to focus the search on the first period, as the gradient of $\omega_{x}^{2}$ reinforce the search on the central part of the diffusion. The behavior of the first resource is more surprising. The most important detections occur at the first periods ( 82 and 2.8 for period 1 and 2), but there is again detection ( 15 for period 7 ) at the end. There is two explanation of this fact. First, a conflict occurs between the target spread and the gradient of $\omega_{x}^{1}$. The first one tends to enforce the detection at the beginning of the movement, while the second enhances the detection when target approaches to the down-right bottom. Second, and that is a crucial point, the second resource spreads the splitting of the first one, since it reinforces detection occurring at the beginning, the middle and the final periods. It is also remarkable that the search areas of the different resources are well distinct and complementary. Their locations depend on the gradient of $\omega_{x}^{\rho}$ (down-right for the first type and down-left for the second). Finally, we still 
recognize surrounding strategy on the first periods, although it is shared between the two resources.

\subsubsection{Multiple modes}

In the following example we shall consider non renewable resources of the same type ( $A=A_{N R}$ and $N=100$ ), which can act under two different detection modes. In first mode $(\rho=1)$ the exponential parameter decreases from the down-right bottom (last picture of figure 8), while in the second one $(\rho=2)$ the exponential parameter decreases from the down-left bottom (last picture of figure 9 ).

\begin{tabular}{|c|c|c|c|c|c|c|c|c|c|c|}
\hline$A$ & $N$ \\
\hline \hline$A_{N R}$ & 100 \\
\hline 1 & $\phi_{1}^{\rho}$ & $\phi_{2}^{\rho}$ & $\phi_{3}^{\rho}$ & $\phi_{4}^{\rho}$ & $\phi_{5}^{\rho}$ & $\phi_{6}^{\rho}$ & $\phi_{7}^{\rho}$ & Figure \\
\hline 1 & 0 & 0 & 0 & 0 & 0 & 0 & 10.9 & 8 \\
\hline 2 & 79.2 & 9.2 & 0.7 & 0 & 0 & 0 & 0 & 9 \\
\hline
\end{tabular}

Table 4: Multi-mode resource.

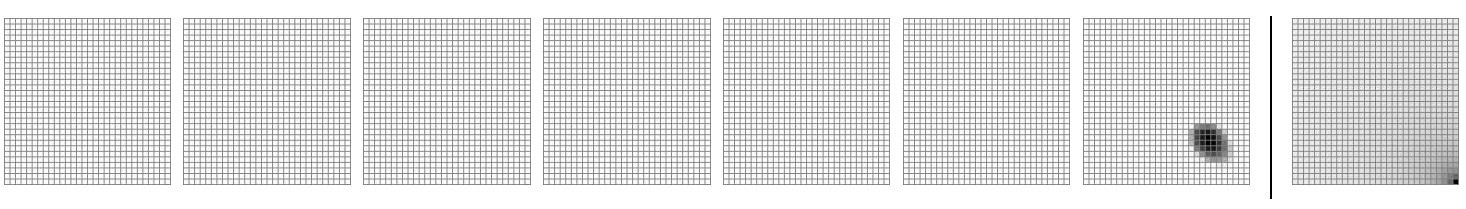

Figure 8: Non renewable resource; $A=A_{N R}, N=100 ;$ mode 1 .

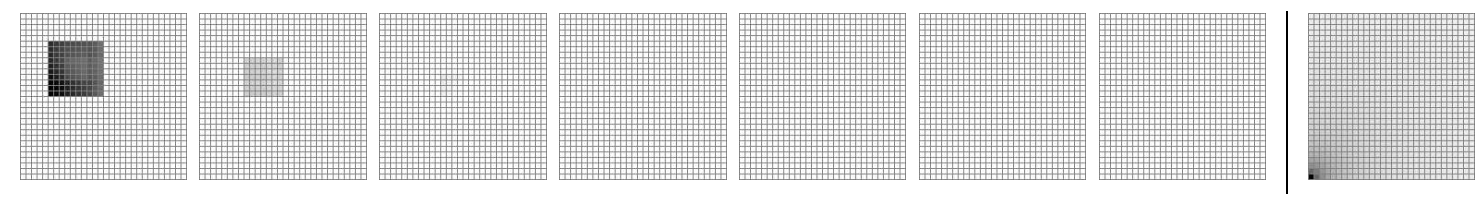

Figure 9: Non renewable resource; $A=A_{N R}, N=100 ;$ mode 2 .

The optimal splitting of the two modes is presented in table 4 . Remark that the sum of all variables $\phi_{k}^{\rho}$ is equal to $N=100$. Search resources are mostly used at the beginning of the target spread, and, since there $\omega^{1}<\omega^{2}$, essentially involve the second mode of detection $\left(\phi_{1}^{2}=79.2\right.$ and $\left.\phi_{2}^{2}=9.2\right)$. But the target moves to the down-right bottom, where the first mode is more powerful. That permits some 
detection at the end of the movement, using first mode $\left(\phi_{7}^{1}=10.9\right)$. Figure 8 and figure 9 represent the spatial sharing of search resource between mode 1 and mode 2. Some surrounding strategy is seen again.

\subsection{Inequality constraints}

The first examples presented here concern a diffusion movement, on 6 periods, with a spread from the starting position followed by a convergence toward the final position. The target distribution is:

$$
\alpha(\vec{x})=Z \times s\left(x_{1}\right)\left(\prod_{k=1}^{5} m\left(x_{k+1}-x_{k}\right)\right) f\left(x_{6}\right)
$$

Again, the non detection functions are uniform $\left(p_{k, x}=\pi\right)$. Moreover, the functions $f$ and $s$ are symmetric and the constraint matrices and vectors, we will use, preserve this symmetry. As a consequence of these facts, the optimization problems we consider here become themselves symmetric. Assuming that the optimal solution $\varphi$ is unique, the effort densities $\varphi_{1}, \varphi_{2}$ and $\varphi_{3}$ are symmetric to $\varphi_{6}, \varphi_{5}$ and $\varphi_{4}$ respectively, for a given problem; so that $\phi_{1}=\phi_{6}, \phi_{2}=\phi_{5}$ and $\phi_{3}=\phi_{4}$. This property will be verified on the four examples described by table 5 . The first example concerns 1-type resources, which renew after 3 periods $\left(A=A_{R 3}\right)$ under equality constraints. The total amount of resource is 50. The second example is the similar; but, this time, inequality constraints are considered. The third and fourth examples involve non renewable resources $\left(A=A_{N R}\right)$, with same power as previously. Equality and inequality cases are both tested. The total amount of resource (100) makes the problems equivalent to the first and second ones.

\begin{tabular}{|c|c|c|c|c|c|c|c|c|c|c|}
\hline$A$ & inequality & $N$ & $\phi_{1}$ & $\phi_{2}$ & $\phi_{3}$ & $\phi_{4}$ & $\phi_{5}$ & $\phi_{6}$ & $\mathbf{P}_{n d}(\varphi)$ & Figure \\
\hline \hline$A_{R 3}$ & no & 50 & 21.1 & 7.8 & 21.1 & 21.1 & 7.8 & 21.1 & $81 \%$ & 10 \\
\hline$A_{R 3}$ & yes & 50 & 32.3 & 10.2 & 7.5 & 7.5 & 10.2 & 32.3 & $79.9 \%$ & 11 \\
\hline$A_{N R}$ & no & 100 & 32.3 & 10.2 & 7.5 & 7.5 & 10.2 & 32.3 & $79.9 \%$ & 11 \\
\hline$A_{N R}$ & yes & 100 & 32.3 & 10.2 & 7.5 & 7.5 & 10.2 & 32.3 & $79.9 \%$ & 11 \\
\hline
\end{tabular}

Table 5: Inequality compared with equality (constraints).

The results of table 5 show that the best results are identically achieved by the three last examples. This was foreseeable since the symmetry equalizes the freedom of those three last problems. The splitting (decreasing and increasing again: $32.3,10.2$, 
$7.5,7.5,10.2,32.3$ ) is coherent with the nature of the movement (a spread followed by a convergence). We notice that $10.2+7.5+7.5<50$. It is strictly forbidden by the equality constraint with $A_{R 3}$. In the first example, sums of three consecutive $\phi_{k}$ must be equal to 50. Such constraints force to a suboptimal solution, where significant amount of resources (21.1) is put on the middle of the movement (periods of maximal target diffusion). The figures 10 and 11 describe the spatial sharing of the resources. We can see surrounding at the beginning period... as well at the final period! Such final surrounding is somewhat upsetting, since surrounding strategy at final period has no sense. The problem is although symmetric and the results perfectly exact, but, when we introduce a time semantic (which is a non symmetric semantic), final surrounding has another meaning than surrounding strategy. Surrounding strategy toward diffusive targets signifies "forbidding" firstly the escape of peripheral trajectories and then detecting central "surviving" trajectories. On the other hand, when converging movement occurs, central detection has to be done at first, so as to wait the arrival of the far trajectories. A detection on the surrounding is to be done later, so as to detect incoming survivors.

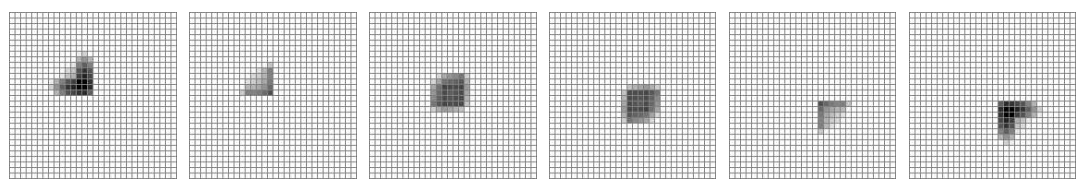

Figure 10: Renewable resource without inequality constraints; $A=A_{R 3}, N=50$.

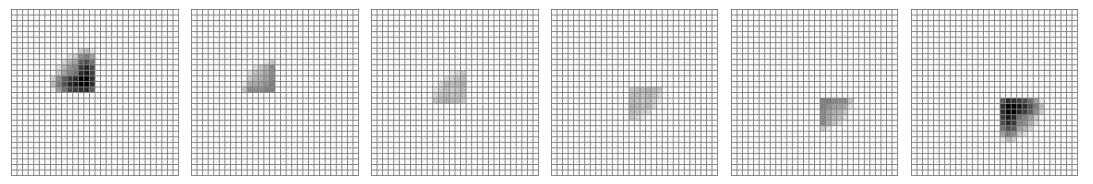

Figure 11: Renewable resource with inequality constraints; $A=A_{R 3}, N=50$.

The earlier example shows that equality constraints is almost as good as inequality constraints ( $81 \%$ versus $79.9 \%$ ). But that is true only when, as in the example, the target spread is very weak. We consider now a final example with a (very) diffusive target. The target distribution comes with start and final positions. Probability on intermediate periods are uniform and all periods are independent:

$$
\alpha(\vec{x})=900^{-4} \times s\left(x_{1}\right) f\left(x_{6}\right)
$$




\begin{tabular}{|c|c|c|c|c|c|c|c|c|c|c|}
\hline$A$ & inequality & $N$ & $\phi_{1}$ & $\phi_{2}$ & $\phi_{3}$ & $\phi_{4}$ & $\phi_{5}$ & $\phi_{6}$ & $\mathbf{P}_{n d}(\varphi)$ & Figure \\
\hline \hline$A_{R 3}$ & no & 50 & 45 & 0 & 5 & 45 & 0 & 5 & $75.7 \%$ & 12 \\
\hline$A_{R 3}$ & yes & 50 & 50 & 0 & 0 & 0 & 0 & 50 & $60.7 \%$ & 13 \\
\hline
\end{tabular}

Table 6: Inequality compared with equality: large target spread.

Optimal solutions are presented in table 6 and the associated figures (the asymmetry of the first result is caused by the non unicity of the solution). The more striking point of these results is the great difference between the two probabilities $(75.7 \%$ versus $60.7 \%$ ). In term of detection probability, it gives $23.3 \%$ versus $39.3 \%$. Improvement is thus considerable.
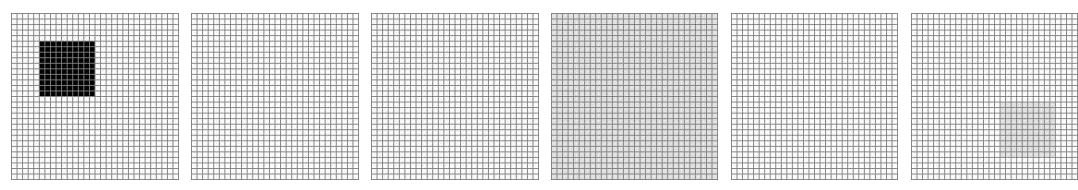

Figure 12: Large target spread, without inequality constraints; $A=A_{R 3}, N=50$.
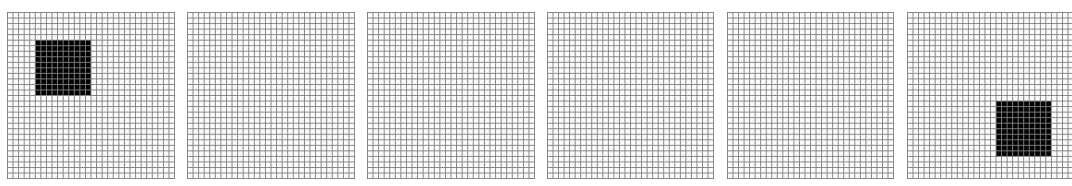

Figure 13: Large target spread, with inequality constraints; $A=A_{R 3}, N=50$.

\section{Conclusion}

Our aim was to solve the problem of spatial and temporal sharing of (possibly) renewable resources. In order to develop feasible optimization methods, the formalism and the algorithm of Brown-de Guenin has played again a central role, allowing us to obtain a whole variety of algorithms, solving problems of increasing difficulty. These algorithms are robust (convergence ensured) and fast since computation requirements are of the same order than Brown's one. Moreover, they seem sufficiently general to 
handle numerous problems of sensor and resource managements arising in complex systems of detection (e.g. sonar, radar, infrared), involving various types of sensors and operating modes. These points have been considered in a general setting. For specific applications, more work has to be done; however our approach seems sufficiently open and versatile to deal with a variety of practical search problems.

\section{References}

[1] B.O. Koopman, Search and Screening: General Principle with Historical Applications. Pergamon Press, New York, 1980.

[2] L.D. Stone, What's Happened in Search Theory Since the 1975 Lanchester Prize?. Operations Research, vol.-37, no 3, May-June 1989, pp. 501-506.

[3] H.R. Richardson, Search Theory. Encyclopaedia of Statistical Science, pp. $314-321$.

[4] S.J. Benkovski, M.G. Monticino and J.R. Weisinger, A Survey of the Search Theory Literature. NAVAl Research Logistics, vol.-38, pp. 469-491, 1991.

[5] L.D. Stone, Theory of Optimal Search, 2-nd ed. . Operations Research Society of America, Arlington, VA, 1989.

[6] K. IIDA, Studies on the Optimal Search Plan. Lecture Notes in Statistics, vol. 70, Springer-Verlag, 1992.

[7] A.R. Washburn, Search and Detection, 2-nd ed. . Operations Research Society of America, Arlington, VA, 1989.

[8] Operations Analysis Study Group (United States Naval Academy), Naval Operations Analysis. Naval Institute Press, Annapolis, Maryland, 1977.

[9] J. de Guenin, Optimum Distribution of Effort: an Extension of the Koopman Basic Theory. Operations Research 9, pp 1-7, 1961.

[10] S.S. Brown, Optimal Search for a Moving Target in Discrete Time and Space. Operations Research 28, pp 1275-1289, 1980.

[11] A.R. Washburn, Search for a moving Target: The FAB algorithm. Operations Research 31, pp 739-751, 1983. 
[12] T. Ibaraki and N. Katoh, Resource Allocation Problems: Algorithmic Approaches. MIT Press, 1988.

[13] L.D. Stone and H.R. Richardson, Search for Targets with Conditionnally Deterministic Motion. SIAM J. of Applied Mathematics, vol. 27, n², pp 239$255,1974$.

\section{A Algorithm for search involving mixed resources}

Rewriting equation (74) as below:

$$
\begin{gathered}
\left(\left.\int_{E} \varphi_{k}^{\rho}(x) d x\right|_{1 \leq k \leq T}\right)_{1 \leq \rho \leq r}=\Phi \\
\boldsymbol{A} \Phi=\Psi
\end{gathered}
$$

we can deduce again:

$$
\Phi=\Phi_{0}+\widetilde{\boldsymbol{A}} \boldsymbol{\nu}
$$

We will denote $\Phi_{k}^{\rho}$ the components of vector $\Phi$.

The new algorithm will act as before; choosing simultaneously a variation $d \Phi=\boldsymbol{A}^{\sim} d \boldsymbol{\nu}$ of $\Phi$ optimizing $d \min _{\varphi} \mathbf{P}_{n d}(\varphi)$ and running a de Guenin's process for each function $\varphi_{k}^{\rho}$. FAB principle will still be used for computing integrals. It is nevertheless necessary to update vector $\mathbf{V}$ for the optimal choice of $d \boldsymbol{\nu}$. The presentation of following results is voluntary concise (details are omitted).

\section{A.1 Optimality conditions}

Let $\kappa$ and $\varrho$ be two particular value of $k$ and $\rho$ indexes. Defining:

$\beta_{\kappa, \varrho}^{\varphi}\left(x_{\kappa}\right)=\left(\prod_{1 \leq \rho \leq r}^{\rho \neq \varrho} p_{\kappa, x_{\kappa}}^{\rho}\left(\varphi_{\kappa}^{\rho}\left(x_{\kappa}\right)\right)\right) \int_{E^{T-1}} \alpha(\vec{x}) \prod_{1 \leq k \leq T}^{k \neq \kappa}\left(\left(\prod_{\rho=1}^{r} p_{k, x_{k}}^{\rho}\left(\varphi_{k}^{\rho}\left(x_{k}\right)\right)\right) d x_{k}\right)$

we obtain:

$$
\mathbf{P}_{n d}(\varphi)=\int_{E} \beta_{\kappa, \varrho}^{\varphi}\left(x_{\kappa}\right) p_{\kappa, x_{\kappa}}^{\varrho}\left(\varphi_{\kappa}^{\varrho}\left(x_{\kappa}\right)\right) d x_{\kappa}
$$


When the search efforts are fixed for all indexes $(k, \rho)$ except for the index $(\kappa, \varrho)$, the problem is in the de Guenin' optimization scheme i.e. :

$$
\begin{aligned}
& \text { Minimize: } P_{n d}\left(\varphi_{\kappa}^{\varrho}\right)=\int_{E} \beta_{\kappa, \varrho}^{\varphi}(x) p_{\kappa, x}^{\varrho}\left(\varphi_{\kappa}^{\varrho}(x)\right) d x, \\
& \text { subject to: } \int_{E} \varphi_{\kappa}^{\varrho}(x) d x=\Phi_{\kappa}^{\varrho} \text { and } \varphi_{\kappa}^{\varrho} \geq 0 .
\end{aligned}
$$

This optimization yields the following conditions of de Guenin:

$$
\left\{\begin{array}{l}
\beta_{\kappa, \varrho}^{\varphi}(x) p_{\kappa, x}^{\varrho}\left(\varphi_{\kappa}^{\varrho}(x)\right)=\eta_{\kappa}^{\varrho} \text { if } \beta_{\kappa, \varrho}^{\varphi}(x)>\eta_{\kappa}^{\varrho} / p_{\kappa, x}^{\varrho}{ }^{\prime}(0) \\
\varphi_{\kappa}^{\varrho}(x)=0 \text { else }
\end{array}\right.
$$

\section{A.2 Choice of $\Delta \nu$}

Subject to $X_{k, \rho}^{\varphi} \triangleq\left\{x \in E / \varphi_{k}^{\rho}(x)>0\right\}$, vector $\mathbf{V}$ is now defined by:

$$
\left\{\begin{array}{l}
\mathbf{V}_{k}^{\rho}=\frac{\int_{X_{k, \rho}^{\varphi}} \frac{p_{k, x}^{\rho}{ }^{\prime}\left(\varphi_{k}^{\rho}(x)\right)}{p_{k, x}^{\rho}{ }^{\prime \prime}\left(\varphi_{k}^{\rho}(x)\right)} d x}{\int_{X_{k, \rho}^{\varphi}} \frac{d x}{\beta_{k, \rho}^{\varphi}(x) p_{k, x}^{\rho}{ }^{\prime \prime}\left(\varphi_{k}^{\rho}(x)\right)}} \text { when } \phi_{k}>0, \\
\mathbf{V}_{k}^{\rho}=\eta_{k}^{0} \text { when } \phi_{k}=0 .
\end{array}\right.
$$

Optimal $\Delta \boldsymbol{\nu}$ is obtained again as a result of a simple optimization problem like (60).

$\operatorname{RR~} \mathrm{n}^{\circ} 3815$ 


\section{B Inequality constraints}

As previously seen and adding slack variables, inequality constrained problems revert to consider the following one (with equality constraints):

$$
\begin{aligned}
& \text { Minimize } \mathbf{P}_{n d}(\varphi)=\int_{E^{T}} \alpha(\vec{x}) \prod_{k=1}^{k=T} p_{k, x_{k}}\left(\varphi_{k}\left(x_{k}\right)\right) \prod_{k=1}^{k=T} d x_{k} \\
& \text { subject to }\left(\int_{E} \varphi_{k}(x) d x\right)_{1 \leq k \leq T}=\phi \text { and }\left(A I_{\Theta}\right)\left(\begin{array}{c}
\phi \\
\phi^{p}
\end{array}\right)=\psi \\
& \text { and }\left(\begin{array}{c}
\phi \\
\phi^{p}
\end{array}\right) \geq 0
\end{aligned}
$$

The algorithm is the same as usually, but the fictitious periods $\phi^{p}$ are not directly considered in the algorithm. In the Brown's process only the functions $\varphi$ are obtained by means of the de Guenin algorithm (a function $\varphi^{p}$ should have no sense). The other difference comes from relaxing the parameterization of $\phi$, that is $\phi=\phi_{0}+\left(A I_{\Theta}\right)^{\sim} \nu$. The calculus of vector $\mathbf{V}$ and the optimal variation of $\Delta \nu$ are changed in consequence, yielding:

$$
\left\{\begin{array}{l}
\mathbf{V}_{k}=\frac{\int_{X_{k}^{\varphi}} \frac{p_{k, x}^{\prime}\left(\varphi_{k}(x)\right)}{p_{k, x}^{\prime \prime}\left(\varphi_{k}(x)\right)} d x}{\int_{X_{k}^{\varphi}} \frac{d x}{\beta_{k}^{\varphi}(x) p_{k, x}^{\prime \prime}\left(\varphi_{k}(x)\right)}} \text { when } \phi_{k}>0 \text { and } 1 \leq k \leq T, \\
\mathbf{V}_{k}=\eta_{\kappa}^{0} \text { when } \phi_{k}=0 \text { and } 1 \leq k \leq T, \\
\mathbf{V}_{k}=0 \text { when } T<k \leq T+\Theta .
\end{array}\right.
$$

The optimal $\Delta \nu$ is obtained by solving the following optimization problem:

$$
\begin{array}{ll}
\begin{array}{ll}
\text { Minimize } \\
\text { subject to }
\end{array} & \mathbf{V}^{t}\left(A I_{\Theta}\right)^{\sim} \Delta \nu, \\
& \left(A I_{\Theta}\right)^{\sim} \Delta \nu+\left(\begin{array}{c}
\phi \\
\phi^{p}
\end{array}\right) \geq 0, \\
& \left\|\left(I_{\Theta}\right) A^{\sim} \Delta \nu\right\| \leq \Delta t .
\end{array}
$$




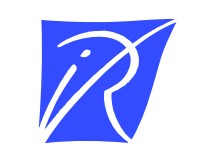

Unité de recherche INRIA Lorraine, Technopôle de Nancy-Brabois, Campus scientifique, 615 rue du Jardin Botanique, BP 101, 54600 VILLERS LÈS NANCY

Unité de recherche INRIA Rennes, Irisa, Campus universitaire de Beaulieu, 35042 RENNES Cedex

Unité de recherche INRIA Rhône-Alpes, 655, avenue de l'Europe, 38330 MONTBONNOT ST MARTIN

Unité de recherche INRIA Rocquencourt, Domaine de Voluceau, Rocquencourt, BP 105, 78153 LE CHESNAY Cedex

Unité de recherche INRIA Sophia-Antipolis, 2004 route des Lucioles, BP 93, 06902 SOPHIA-ANTIPOLIS Cedex

Éditeur

INRIA, Domaine de Voluceau, Rocquencourt, BP 105, 78153 LE CHESNAY Cedex (France)

http://www.inria.fr

ISSN 0249-6399 